\title{
The Role of Nonlinear Body Force in Electrostatic Propeller Driven by Atmospheric DC Corona Discharges
}

Farsahd Sohbatzadeh ( $\nabla$ f.sohbat@umz.ac.ir)

University of Mazandaran

Hamed Soltani Ahmadi

University of Mazandaran

Saeed Mirzanejhad

University of Mazandaran

\section{Research Article}

Keywords: electric thruster, MATLAB software, variable mass systems, COMSOL, wire-cylinder

Posted Date: December 14th, 2021

DOl: https://doi.org/10.21203/rs.3.rs-1122828/v1

License: (c) (i) This work is licensed under a Creative Commons Attribution 4.0 International License.

Read Full License 


\title{
The role of nonlinear body force in electrostatic propeller driven by atmospheric DC corona discharges
}

\author{
F. Sohbatzadeh ${ }^{\mathrm{a}, \mathrm{b}}, *$, H. Soltani Ahmadia, ${ }^{\mathrm{a},}$, H. Mirzanejhad ${ }^{\mathrm{a}, \mathrm{b}}$ \\ a Department of Atomic and Molecular Physics, Faculty of Science, University of Mazandaran, Babolsar, Iran \\ ${ }^{\mathrm{b}}$ Plasma Technology Research Core, Faculty of Science, University of Mazandaran, Babolsar, Iran \\ *Corresponding author: E-mail: f.sohbat@umz.ac.ir
}

\begin{abstract}
In this study, the body force generated by atmospheric positive and negative corona discharges were investigated using a wire-cylinder configuration experimentally and numerically. We provided new insight into the atmospheric electric thruster by introducing a nonlinear term in body force constituent the thrust of the system. It was observed that the direction of both body forces and electric winds is always from the wire to the cylinder irrespective of the applied voltage polarity. It was illustrated that the corresponding thrusts and the electric wind of the positive corona are larger than that of the negative corona discharge. We took into account the nonlinear mechanisms to explain the difference in thrust forces in positive and negative corona discharges. To elucidate the origin of the body force in corona discharges, we performed 2-D simulations via COMSOL Multiphysics and MATLAB software. The results of the numerical simulation showed that in addition to the linear body force (Coulomb force) a strong nonlinear body force is generated around the wire electrode that plays a crucial role in corona thrusters. To verify the direction and magnitude of the thrust, a simple theory was proposed based on variable mass systems and confirmed by published experimental works.
\end{abstract}

\section{Introduction}

Corona discharge occurs when the high electric field is not uniform near the electrodes. In atmospheric pressure, the electric field strength near one or both electrodes should be greater than the rest of the discharge gap. By increasing the applied voltage on the electrodes, corona discharge sets up around the sharp edge until the initiation of the sparks or streamers $[1,2]$. Corona discharge in high-pressure structures and transmission lines is an undesirable and dangerous phenomenon, but a controlled one can create an electric current and wind by charging particles and transferring their momentum to neutral particles, which has many applications in various fields. Studies on the direct current (DC) corona discharge were typically treated in needle-to-plate or wire-to-cylinder configurations. In the wire-to-cylinder setup, both positive and negative coronas are formed around the wire but with different features [3]. In the literature, the positive and negative coronas are distinguished by the polarity of the applied high voltage to the electrode whose surrounding gas is ionized, partially. In the positive corona, the streamers, and in the negative one the Trichel pulses play the main aspects of the process [4-7]. Corona discharge has been theoretically and experimentally studied for its many applications such as electrostatic precipitation, water treatment, air purification, surface treatment, and plasma medicine [8-12]. Current researches on corona discharge covered many fields including plasma actuators for flow control [13-15] and propulsion by electrohydrodynamic (EHD) thrusters [16-23]. The first observation of the EHD current was made in 1709 by Hauksbee [24]. Hauksbee discovered the production of low-velocity 
airflow when the voltage was applied between two electrodes, a phenomenon later referred to by Newton as the "electric wind"[25]. In the late 19 century, Chattock first studied electric wind quantitatively [26]. In 1957, Harney investigated the electrical characteristics of corona discharge and the change in flow aerodynamic parameters to measure the generated force [27]. Davis conducted one of the first numerical studies on corona discharges using the finite element method [28]. In 2008, Larson et al. investigated electrohydrodynamic force in a corona discharge and compared their results with that of experimental works [29]. Colas et al. optimized the corona discharge regime in a five-electrode configuration and measured the wind velocity of up to $10 \mathrm{~m} / \mathrm{s}$ [30]. They also calculated the electrohydrodynamic force using velocity profiles. In an experimental study, Moreau et al. investigated the current-to-thrust conversion with a threeelectrode design. They improved the thruster effectiveness using a proper design. [31]. In another study, Moreau et al. examined the thrust force generated in positive and negative corona discharge by considering the wire-cylinder configuration [32]. An outstanding result was achieved by Xu et al in developing a drone with corona propulsion by applying a DC high voltage of $+40 \mathrm{kV}$ to generate a thrust of $3.2 \mathrm{~N}$ and flew the drone for a distance of $40-45 \mathrm{~m}$ with a duration of $8-9 \mathrm{~s}$ [33].

Although the accepted theoretical models of body force contribute much to understanding the producing corona propulsions, the lack of true knowledge still restrains the popularization and application of such propellers. Therefore, the understanding of the body force mechanisms could facilitate the development of corona propulsion structures. It is generally accepted that the mechanism for electrohydrodynamic force production is based on the Coulomb force, as a linear term, acting on the plasma space charge in an effective electric field. Our previous theoretical work showed that a new mechanism of electrohydrodynamic force could be a nonlinear body force that arose from the gradient in the effective electric intensity inside the discharge zone. This nonlinear term contributes the electric wind generation independent of electric field polarity and charges sign as follows[34]:

$$
\boldsymbol{f}_{V \alpha}=-\frac{3}{8 \pi} \nabla|E|^{2}\left[\sum_{\alpha=e, i} \frac{\omega_{p \alpha}^{2}}{v_{\alpha n}^{2}}\right]
$$

Where $\omega_{p \alpha}$, and $v_{\alpha \mathrm{n}}$ are the angular frequency and effective collision frequency for momentum transfer, respectively. $\alpha$ refers to the electron and ion species, and $\omega_{p \alpha}^{2}=\frac{4 \pi e^{2} n_{\alpha}}{m_{\alpha}}, m_{\alpha}$ and $n_{\alpha}$ are the mass and density of electron and ion, respectively. The effective collision frequency is given by $v_{\alpha n}=N V_{\alpha} \sigma_{t r}$, where $\sigma_{t r}$ is the momentum transfer cross-section, $N$ is the number density of neutral particles and $V_{\alpha}$ is the average velocity of random motion for the particles of type $\alpha$. It is seen that the nonlinear body force pushes all charged species out of the strong electric field zone independent of the sign and electric field polarity.

In this study, we proposed and examined the contribution of a nonlinear body force mechanism in corona propulsions. The contribution of the nonlinear body force arising from a positive and negative corona discharge was simulated in comparison with the linear Coulombic force. Using 
the simulation results, we showed that there is a nonlinear body force, acts through a different mechanism, influencing the discharge assembly around the wire electrode due to a strong gradient in the electric field intensity. It was also shown that the direction of the nonlinear body force in both positive and negative corona discharges is always from the wire to the cylinder. To consider the influence of linear (Coulomb force) and nonlinear body force in wire-cylinder configuration, the results of references [34-36] were used for both coronas. It was demonstrated that during the running of both coronas the electric current can be decomposed into DC and AC signals that characterize the corona process and distinguish positive and negative coronas. To verify the direction and magnitude of the thrust, we introduced a simple theory based on the variable mass force equation with taking into account the examined wind direction. Finally, it was proved that the corona discharge assembly acts as a system of propellers that produces a thrust via a wind generation like a pusher fan on a boat.

This paper is organized as follows: in section 2, materials and methods including simulation procedures, and experimental setup are introduced. In section 3 simulation and experimental results are given, in section 4, a simple theory for corona thrusters are given, and in section 5 and 6 , the discussions and results are given taking into account the linear and nonlinear body forces.

\section{Materials and Methods}

\subsection{Simulation section}

Linear and non-linear body forces in positive and negative corona discharge were investigated using COMSOL Multiphysics (plasma module) and MATLAB software at atmospheric pressure. The linear and nonlinear terms of the body force per unit volume $\left(\mathrm{N} / \mathrm{m}^{3}\right)$ can be written as follows [34]:

$$
\begin{aligned}
& F_{\text {coulomb }}=\rho \times E \\
& F_{\text {nonlinear }}=-\frac{3}{8 \pi} \times \nabla|E|^{2}\left[\sum_{\alpha=e, i} \frac{\omega_{p \alpha}^{2}}{v_{\alpha n}^{2}}\right]
\end{aligned}
$$

In the above equations, $\rho$ is the net charge density of the species $\left(\mathrm{C} . \mathrm{m}^{-3}\right), \mathrm{E}$ is the electric field strength in $V / m$ such that $E=\sqrt{E_{y}^{2}+E_{x}^{2}}$.

As can be seen in figure 1, in a 2-D simulation, two circles act as electrodes with radii of $0.5 \mathrm{~mm}$ and $10 \mathrm{~mm}$ were placed at a distance of $30 \mathrm{~mm}$ from each other. We set the wire and the cylinder as the powered and ground electrodes, respectively. In the simulations, we set the powered electrode (wire) at a DC voltage of $+10 \mathrm{kV}$ and $-10 \mathrm{kV}$ for positive and negative corona, respectively. 

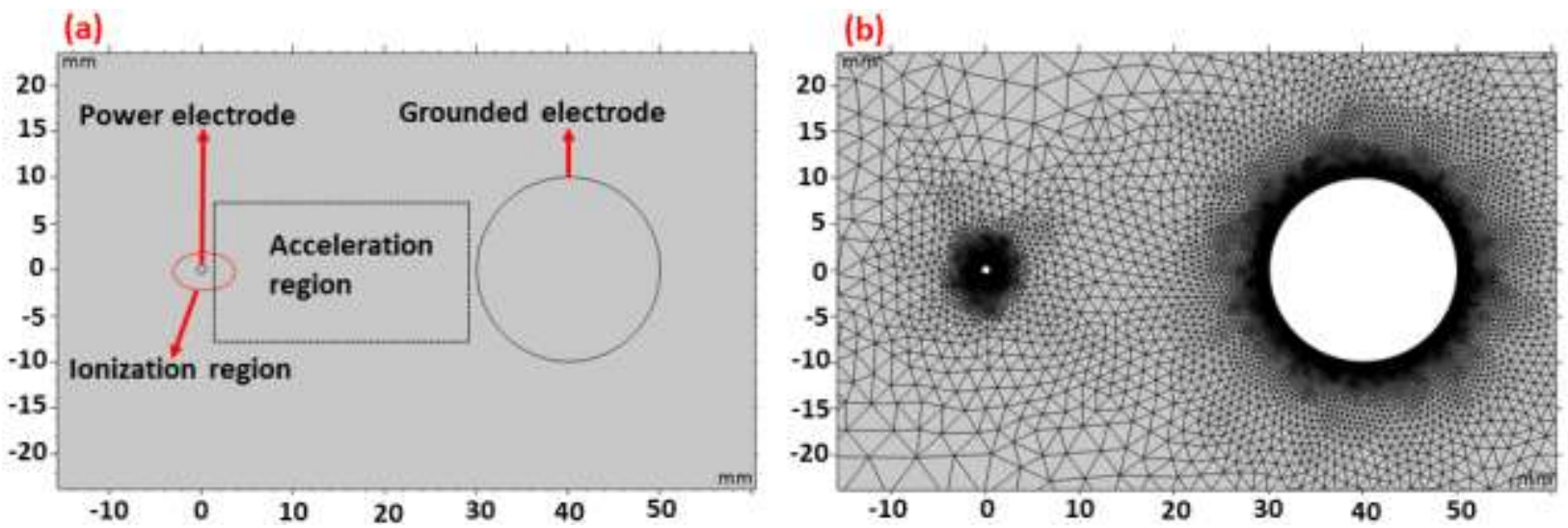

Figure 1. Schematic picture of the: (a) Simulation geometry and (b) meshing.

In this simulation, we took into account the electron impact reactions, reaction between heavy species, and surface reactions including the secondary emission from the electrode surface. As can be seen in table 1, the reactions include electron impact ionization, electron attachment, elastic collisions, excitations, recombination, and ion conversion processes. In table $1, \mathrm{~N}_{2} \mathrm{~S}$ represents $\mathrm{N}_{2}$ $\left(\mathrm{A}^{3} \Sigma\right)$ as the nitrogen excited species and $\mathrm{O}_{2} \mathrm{~S}$ represents $\mathrm{O}_{2}\left(\mathrm{a}^{1} \Delta\right)$ as the singlet oxygen excited species. $\mathrm{O}$ and $\mathrm{N}$ represent $\mathrm{O}\left({ }^{3} \mathrm{P}\right)$ and $\mathrm{N}\left({ }^{4} \mathrm{~S}\right)$, respectively.

Table 1. The dominant reactions in the discharge channel [37-40].

\begin{tabular}{|c|c|c|c|}
\hline Number & Reaction & $\begin{array}{c}\text { Collision } \\
\text { Type }\end{array}$ & $\begin{array}{l}\text { Reaction Rate }{ }^{\mathrm{a}} \text { or } \\
\text { Energy loss }(\mathrm{eV})^{\mathrm{b}}\end{array}$ \\
\hline $\mathbf{R}_{\mathbf{1}}$ & $\mathrm{e}+N_{2} \rightarrow 2 \mathrm{e}+N_{2}^{+}$ & Ionization & Lxcat Data ${ }^{c}$ \\
\hline $\mathbf{R}_{\mathbf{2}}$ & $\mathrm{e}+N_{2} \rightarrow N_{2}^{-}$ & Attachment & Lxcat Data \\
\hline $\mathbf{R}_{\mathbf{3}}$ & $\mathrm{e}+N_{2}+N_{2} \rightarrow N_{2}+N_{2}^{-}$ & Attachment & Lxcat Data \\
\hline $\mathbf{R}_{4}$ & $\mathrm{e}+N_{2} \rightarrow \mathrm{e}+N_{2}$ & Elastic & Lxcat Data \\
\hline $\mathbf{R}_{5}$ & $\mathrm{e}+N_{2} S \rightarrow \mathrm{e}+N_{2} S$ & Excitation & Lxcat Data \\
\hline $\mathbf{R}_{6}$ & $\mathrm{e}+N_{2} \rightarrow e+N+N$ & Excitation & Lxcat Data \\
\hline $\mathbf{R}_{7}$ & $\mathrm{e}+\mathrm{O}_{2} \rightarrow \mathrm{e}+\mathrm{O}_{2}$ & Elastic & Lxcat Data \\
\hline $\mathbf{R}_{8}$ & $\mathrm{e}+\mathrm{O}_{2} \rightarrow O+O^{-}$ & Attachment & Lxcat Data \\
\hline $\mathbf{R}_{\mathbf{9}}$ & $\mathrm{e}+\mathrm{O}_{2} \mathrm{~s} \rightarrow \mathrm{e}+\mathrm{O}_{2} \mathrm{~s}$ & Excitation & Lxcat Data \\
\hline $\mathbf{R}_{10}$ & $\mathrm{e}+O_{2} \rightarrow e+O+O$ & Excitation & Lxcat Data \\
\hline $\mathbf{R}_{11}$ & $\mathrm{e}+\mathrm{O}_{2} \rightarrow 2 \mathrm{e}+\mathrm{O}_{2}^{+}$ & Ionization & Lxcat Data \\
\hline $\mathbf{R}_{12}$ & $+N_{2}^{-} \rightarrow 2 N_{2} N_{2}^{+}$ & Recombination & $1.2 \times 10^{12}$ \\
\hline $\mathbf{R}_{13}$ & $\mathrm{O}+\mathrm{O}_{2}+\mathrm{O}_{2} \rightarrow \mathrm{O}_{2}+\mathrm{O}_{3}$ & Reaction & 199.7 \\
\hline $\mathbf{R}_{\mathbf{1 4}}$ & $\mathrm{O}+\mathrm{N}_{2}+\mathrm{O}_{2} \rightarrow \mathrm{N}_{2}+\mathrm{O}_{3}$ & Reaction & 199.7 \\
\hline $\mathbf{R}_{15}$ & $\mathrm{O}+\mathrm{O}_{3} \rightarrow \mathrm{O}_{2}+\mathrm{O}_{2}$ & Reaction & $4.7 \times 10^{3}$ \\
\hline $\mathbf{R}_{16}$ & $\mathrm{e}+\mathrm{O}_{2}+\mathrm{O}_{2} \rightarrow \mathrm{O}_{2}+\mathrm{O}_{2}^{-}$ & Attachment & $3.6 \times 10^{3}$ \\
\hline $\mathbf{R}_{17}$ & $\mathrm{e}+\mathrm{O}_{2}+N_{2} \rightarrow N_{2}+O_{2}^{-}$ & Attachment & \\
\hline $\mathbf{R}_{\mathbf{1 8}}$ & $+N_{2}+O_{2} \rightarrow N_{4}^{+}+O_{2} N_{2}^{+}$ & Ion conversion & $3.6 \times 10^{3}$ \\
\hline $\mathbf{R}_{19}$ & $+O_{2} \rightarrow 2 N_{2}+O_{2}^{+} N_{4}^{+}$ & Ion conversion & $1.8 \times 10^{7}$ \\
\hline $\mathbf{R}_{\mathbf{2 0}}$ & $+N_{2}+N_{2} \rightarrow N_{4}^{+}+N_{2} N_{2}^{+}$ & Ion conversion & $2.3 \times 10^{8}$ \\
\hline $\mathbf{R}_{\mathbf{2 1}}$ & $+N_{2}+O_{2} \rightarrow O_{4}^{+}+N_{2} O_{2}^{+}$ & Ion conversion & $1.8 \times 10^{7}$ \\
\hline $\mathbf{R}_{22}$ & $+\mathrm{O}_{2}+\mathrm{O}_{2} \rightarrow \mathrm{O}_{4}^{+}+\mathrm{O}_{2} \mathrm{O}_{2}^{+}$ & Ion conversion & $1.5 \times 10^{10}$ \\
\hline
\end{tabular}




$$
\begin{aligned}
& \begin{array}{lclc}
\mathbf{R}_{23} & +\mathrm{O}_{2}^{-} \rightarrow 3 \mathrm{O}_{2} \mathrm{O}_{4}^{+} & \text {Recombination } & 1.5 \times 10^{10} \\
\mathbf{R}_{24} & +\mathrm{O}_{2}^{-} \rightarrow \mathrm{N}_{2}+3 \mathrm{O}_{2} \mathrm{~N}_{2}+\mathrm{O}_{4}^{+} & \text {Recombination } & 6 \times 10^{10} \\
\mathbf{R}_{25} & +\mathrm{e} \rightarrow 2 \mathrm{O}_{2} \mathrm{O}_{4}^{+} & \text {Recombination } & 7.2 \times 10^{10} \\
& & & \frac{8.2 \times 10^{11} \times(300)^{0.5}}{T_{e}}
\end{array} \\
& \text { R26 }+\mathrm{e} \rightarrow 2 \mathrm{O}_{2} \mathrm{O}_{2}^{+} \\
& \text {Recombination } \frac{1.5 \times 10^{9} \times(300)}{T_{e}}
\end{aligned}
$$

${ }^{a}$ Units are $\frac{\mathrm{m}^{3}}{\mathrm{s.mol}}$ for two-body reactions and $\frac{\mathrm{m}^{6}}{\mathrm{s. \textrm {mol } ^ { 2 }}}$ for three-body reactions.

${ }^{\mathrm{b}}$ Energy loss for electron impact reaction in Lxcat database.

${ }^{\mathrm{c}}$ Database of electron collisions with heavy species considering the cross-section of the collision.

${ }^{*} T_{e}$ is the electron temperature and in the unit of $\mathrm{K}$.

Because the COMSOL software cannot solve higher derivatives in the plasma module algorithm, we calculated the value of the nonlinear body force using MATLAB software based on the COMSOL outputs. For this purpose, as depicted in figure 2, we divided the space between the two electrodes into 2601 points and formed $51 \times 51$ matrices. At each point, we calculated all needed parameters for evaluating the nonlinear body force term including charged species density, electric field distribution, neutral density and averaged velocity of the $\alpha$-type particles. The results of linear and nonlinear terms will be given in the result section. Figure 3 shows the flowchart of the simulation procedures that were used in this work.

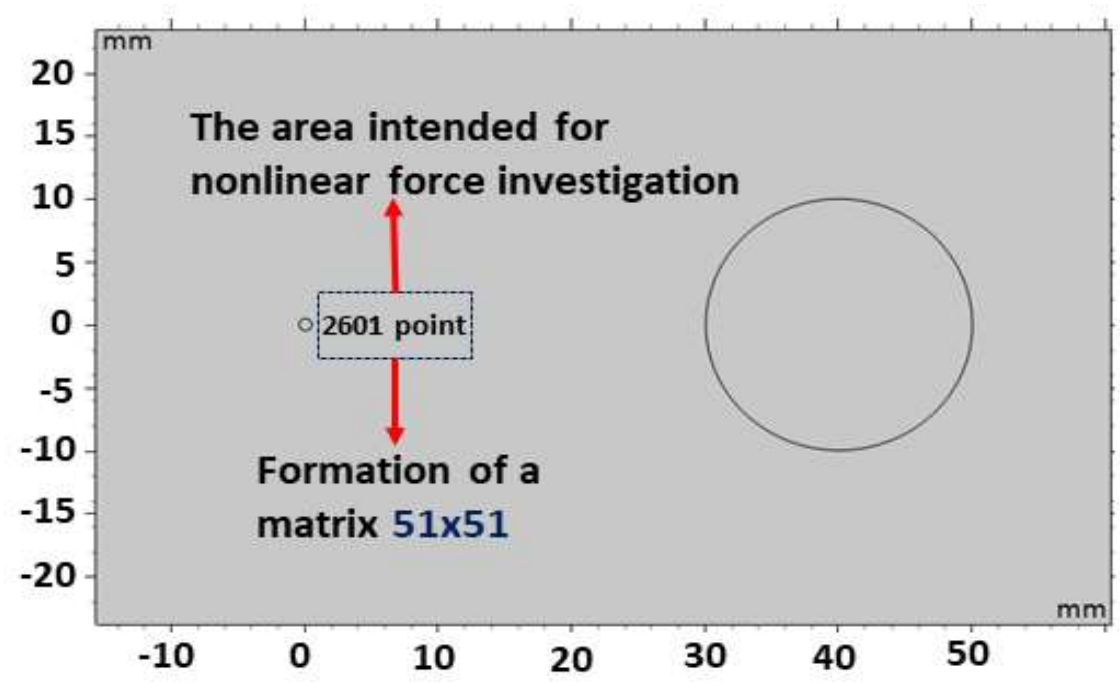

Figure 2. A matrix of $51 \times 51$ near the wire, where the nonlinear body force is significant. 


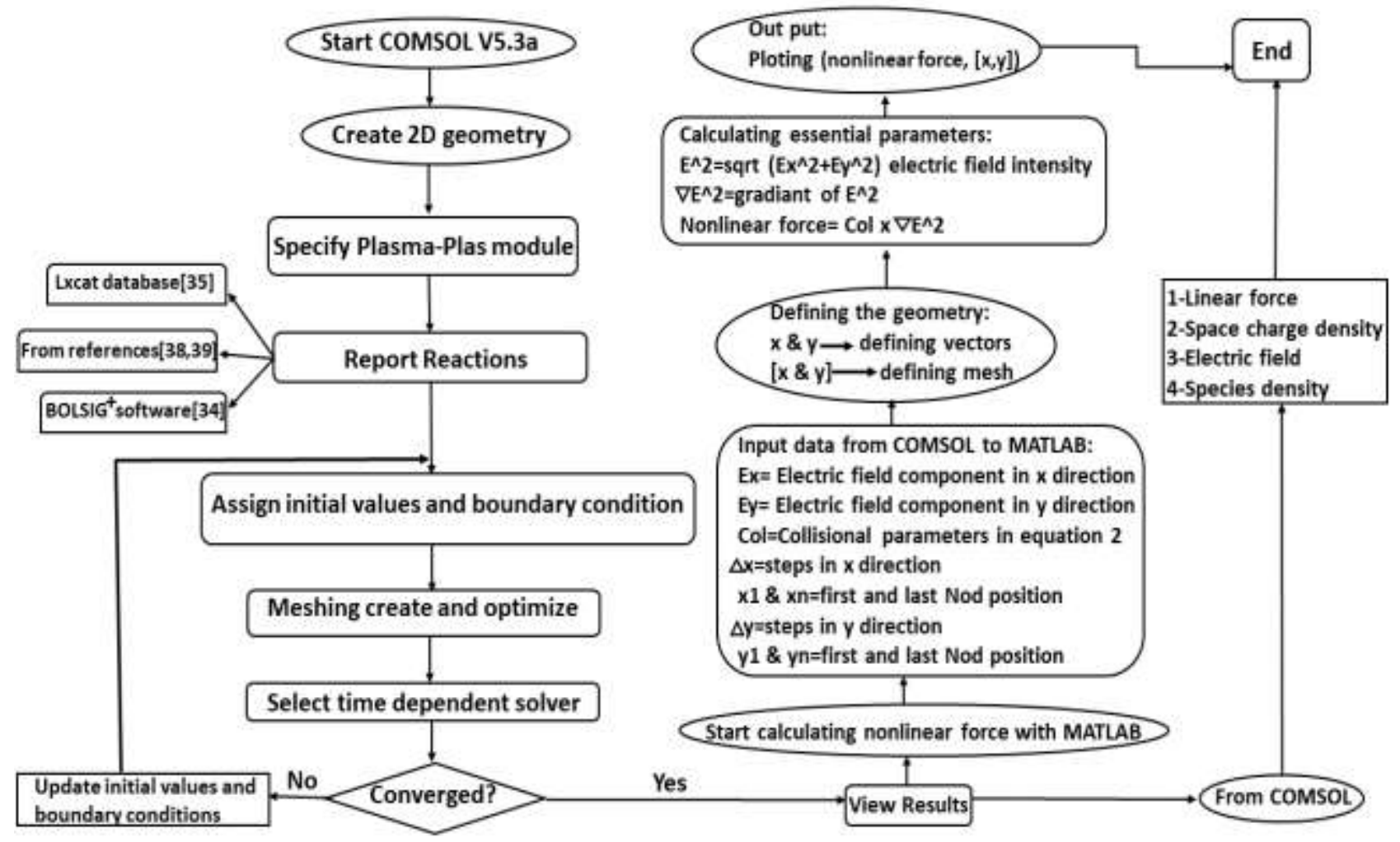

Figure 3. Flowchart of the simulation procedures.

\subsection{DC high voltage power supply and voltage-current measurement}

Positive and negative DC high voltage power supplies $(0- \pm 30 \mathrm{kV}, 3 \mathrm{~mA}, 90 \mathrm{~W})$ were made using a two-stage full-wave Cockcroft-Walton voltage multiplier. The input for the Cockcroft-Walton module was a sine wave high voltage with frequency and peak to peak values of $7.2 \mathrm{kHz}$ and 22 $\mathrm{kV}$, respectively. The DC applied voltage with a resolution of $0.1 \mathrm{kV}$ was adjusted manually. The applied voltage to the wire electrode was measured using a high voltage probe (Tektronix P6015A.), and the current signals were monitored by a high-precision analog multimeter (Leybold Demo-Multimeter 53,191, precision $\pm 0.05 \mu \mathrm{A}$ ). All signals were recorded by a four-channel digital oscilloscope (GW Instek GDS-3354). To run the setup in corona mode, a copper wire electrode having $20 \mathrm{~cm}$ length and $0.1 \mathrm{~mm}$ diameter, together with a cylindrical electrode with $20 \mathrm{~cm}$ long and $10 \mathrm{~mm}$ diameter were used. According to figure 4, both electrodes were held by an insulating housing (No. 1 in Figure 4(a)) and were placed at a distance of $3 \mathrm{~cm}$ from each other. In each experiment, the applied voltage was last for 30 seconds. 
(a)

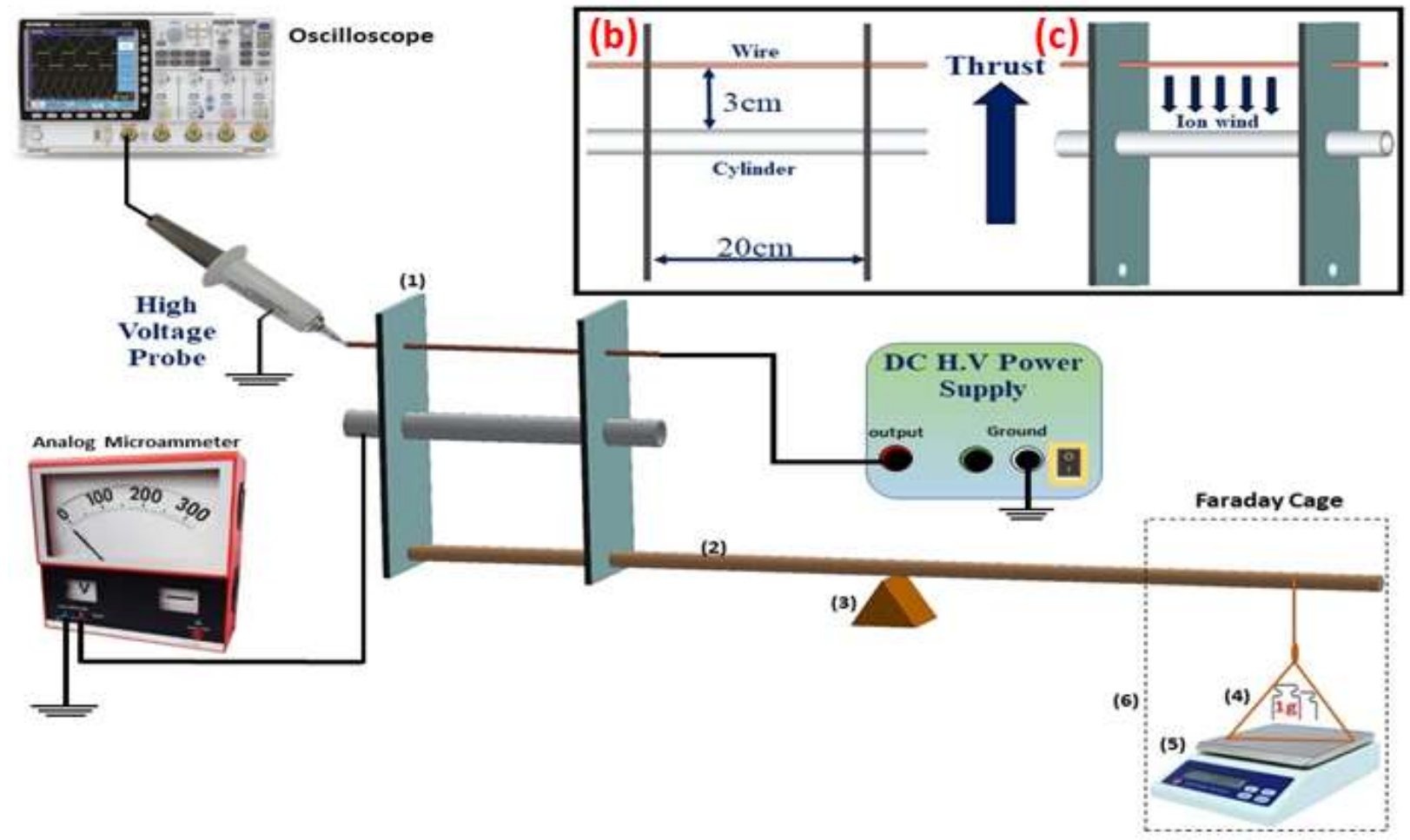

Figure 4. Sketch of the thrust measurement setup. (a): layout of the static equilibrium condition. (1) corona assembly, (2) metal beam, (3) knife-edge support, (4) weights, (5) digital scale, (6) Faraday Cage (b): cross-section of the corona assembly (c): ion wind and the corresponding thrust direction

\subsection{Thrust force measurement}

The experimental setup for thrust measurement was depicted in figures 4(a), (b), and (c). The cross-section of the corona assembly and the direction of the ion wind were shown in figures (b) and (c), respectively. A rigid metal beam (No. 2 in Figure 4(a)) was supported by a knife-edge (No. 3 in Figure 4(a)) and kept in static equilibrium by hanging five masses of one gram via a thread in one hand (No. 4)) and putting the corona assembly on the other hand (No. 1 in Figure 4(a)). To examine the balance of the beam under the corona running, a digital scale with an accuracy of $0.01 \mathrm{gr}$ (No. 5 in Figure 4(a)) was used. To protect it from electromagnetic noises, a Faraday cage was used (No. 6 in Figure 4(a)). To measure the thrust force of the coronas, the calibration of the setup was necessary to avoid the significant errors arising from the weight forces of the metal beam arms. To do that, we made initially artificial thrusts by hanging several weights on the corona assembly position. Then we measured the change in the reaction of the digital scale. We obtained the artificial thrust of the corona assembly by this method, then we applied the 
calibration curve for measuring the actual thrust of the coronas. It was proved that the thrust measurement setup has a linear trend as shown in figure 5. The thrust of the corona was obtained by $f_{b}=0.3004 X+6 \times 10^{-5}$, is the change in digital scale in Newton.

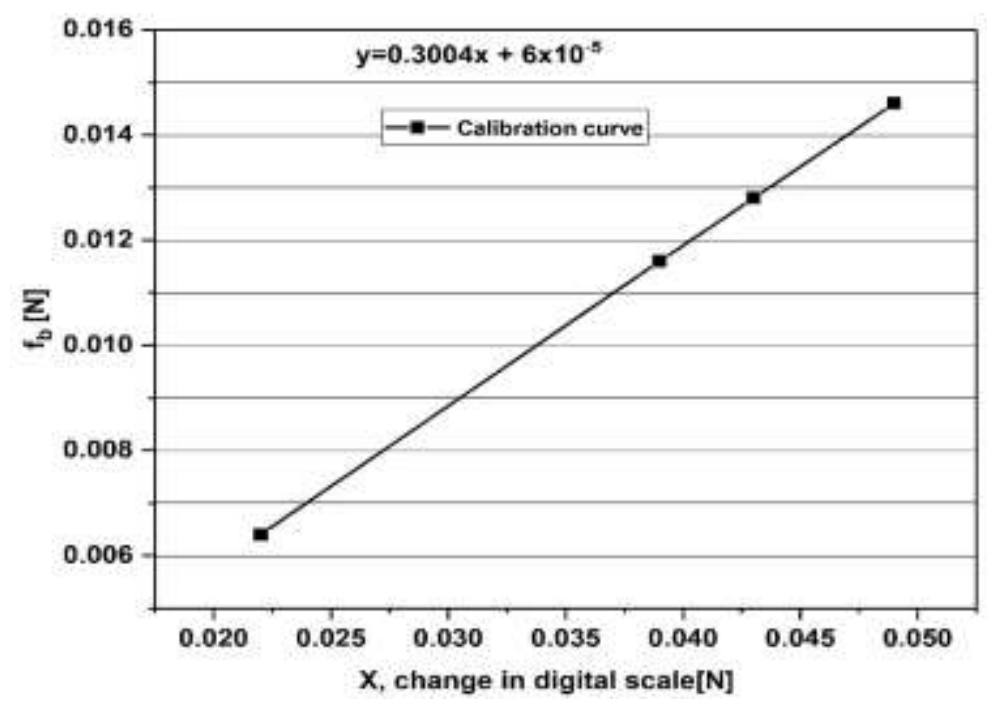

Figure 5. Calibration curve for the thrust measurement

By running the coronas in a variety of electrical parameters, we recorded the change in the digital scale, then converted the change into the thrust force by $\mathrm{T}=0.3004 \mathrm{X}+6 \times 10^{-5}$, where $X=\Delta m \times$ $9.80665 / 1000$ is the change in the digital scale in Newton. The accuracy of thrust measurement was evaluated to be $\pm 0.04 \mathrm{mN}$.

The magnitudes of the consumed electrical power and thrust effectiveness were calculated according to the following equations, respectively [32]:

$$
\begin{aligned}
& \mathrm{P}=\mathrm{V} \times \mathrm{I} \\
& \theta=\frac{\mathrm{T}}{\mathrm{P}}
\end{aligned}
$$

Where T represents the thrust force, $\mathrm{P}$ is the consumed electric power, I the electric current, and $\theta$ the thrust effectiveness.

\subsection{Electric wind velocity measurement}

The average velocity of the electric wind was measured using a Pitot tube which was connected to a micro manometer (CEM, DT-8920. PONPE, Inc.). The stainless steel Pitot tube might lead to 
sparking at distances near the wire electrode, then a homemade glass capillary tube with an inner diameter of $0.5 \mathrm{~mm}$ was used to measure the total pressure. Also, static pressure was calculated by the static pressure tube near the site. As depicted in figures 6(a) and (b), a 1-D y-positioner with $0.1 \mathrm{~mm}$ accuracy was employed to displace the glass tube along the horizontal axes. With this method, the vertical velocity of the electric wind was measured with an accuracy of $\pm 0.01 \mathrm{~m} / \mathrm{s}$.

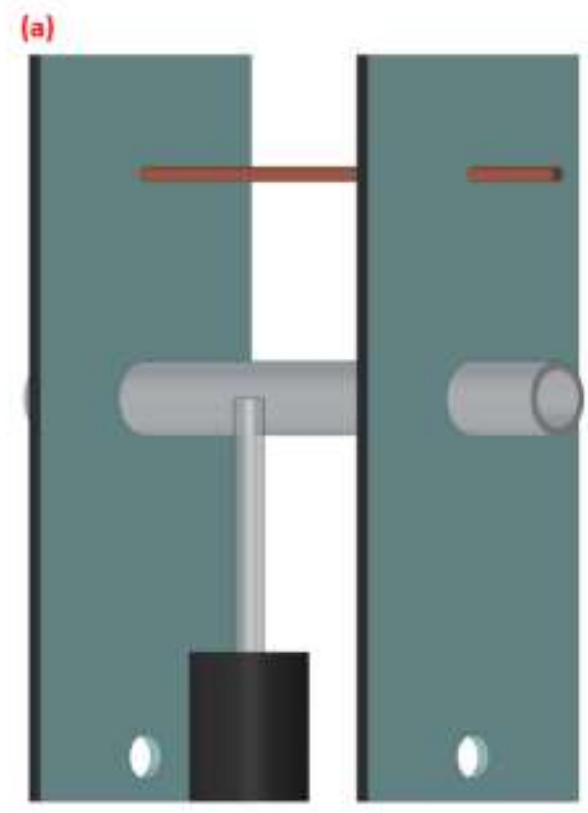

(b)

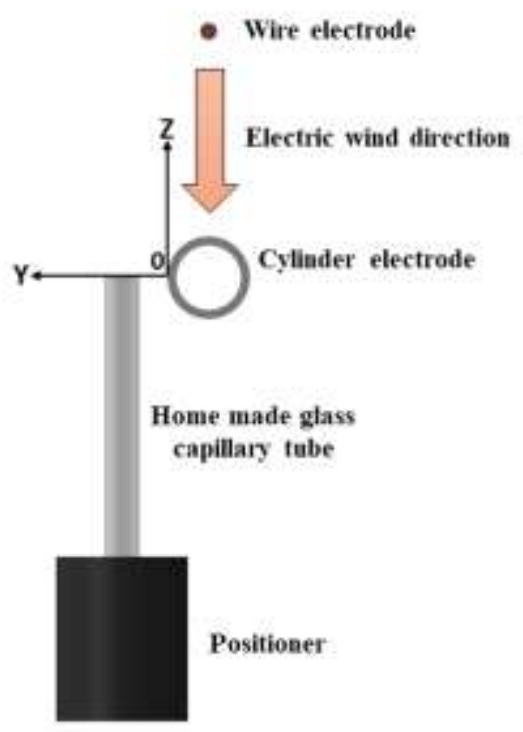

Figure 6. Sketch of the electric wind vertical velocity measurement with the glass capillary tube: (a) front view and (b) side view.

\subsection{Electric wind direction test (EWD test)}

As depicted in figures 7(a) and (b), three incense sticks with equal lengths were installed parallel in the space between the two electrodes. The tip of each incense was set adjacent to the wire electrode and in the middle of the cylinder electrode. The observations of this experiment showed that the direction of the moving smoke changes due to the production of the electric wind. It was demonstrated that the upward smoke of the incense in corona-off condition changes to be downward with applying both positive and negative high voltage to the wire. It was also visualized that the electric wind is initiated from the powered wire zone rather than the cylinder. This claim was proved by placing five parallel incense sticks in the corona gap with stair configuration which showed that the change in smoke direction takes place in sequence from the nearest incense to the wire (figure 7 b). All images were recorded using the digital camera (SONY DSC-HX200 V). 

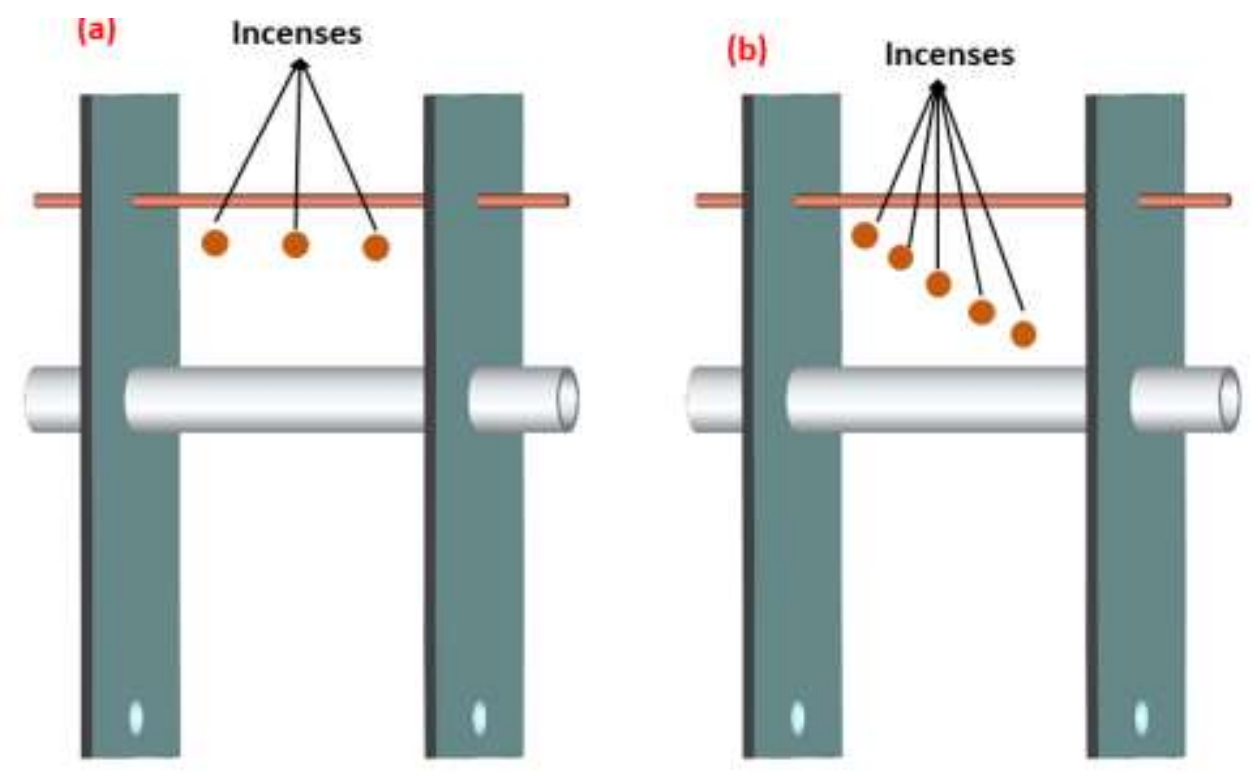

Figure 7. Sketch of the incenses between the electrodes: (a) at the same distance from the wire (b) at different distances from the wire

\section{Results}

\subsection{Simulation Results}

By applying a voltage of $\pm 10 \mathrm{kV}$ to the wire, a corona discharge was produced while the cylinder electrode was connected to the ground. According to the characteristics time of the ionization and attachment processes, we conducted the simulation up to $10 \mathrm{~ns}$ with a time step of 10 ps. The distribution of the electric field vector was shown by figures 8(a) and (b) for positive corona on 10 ns, and that of the negative corona by figures 8 (c) and (d). It is seen that the electric field has a maximum magnitude of $4 \times 10^{6} \mathrm{~V} / \mathrm{m}$ and $2.5 \times 10^{6} \mathrm{~V} / \mathrm{m}$ around the wire electrode for both negative and positive corona discharges, respectively. 

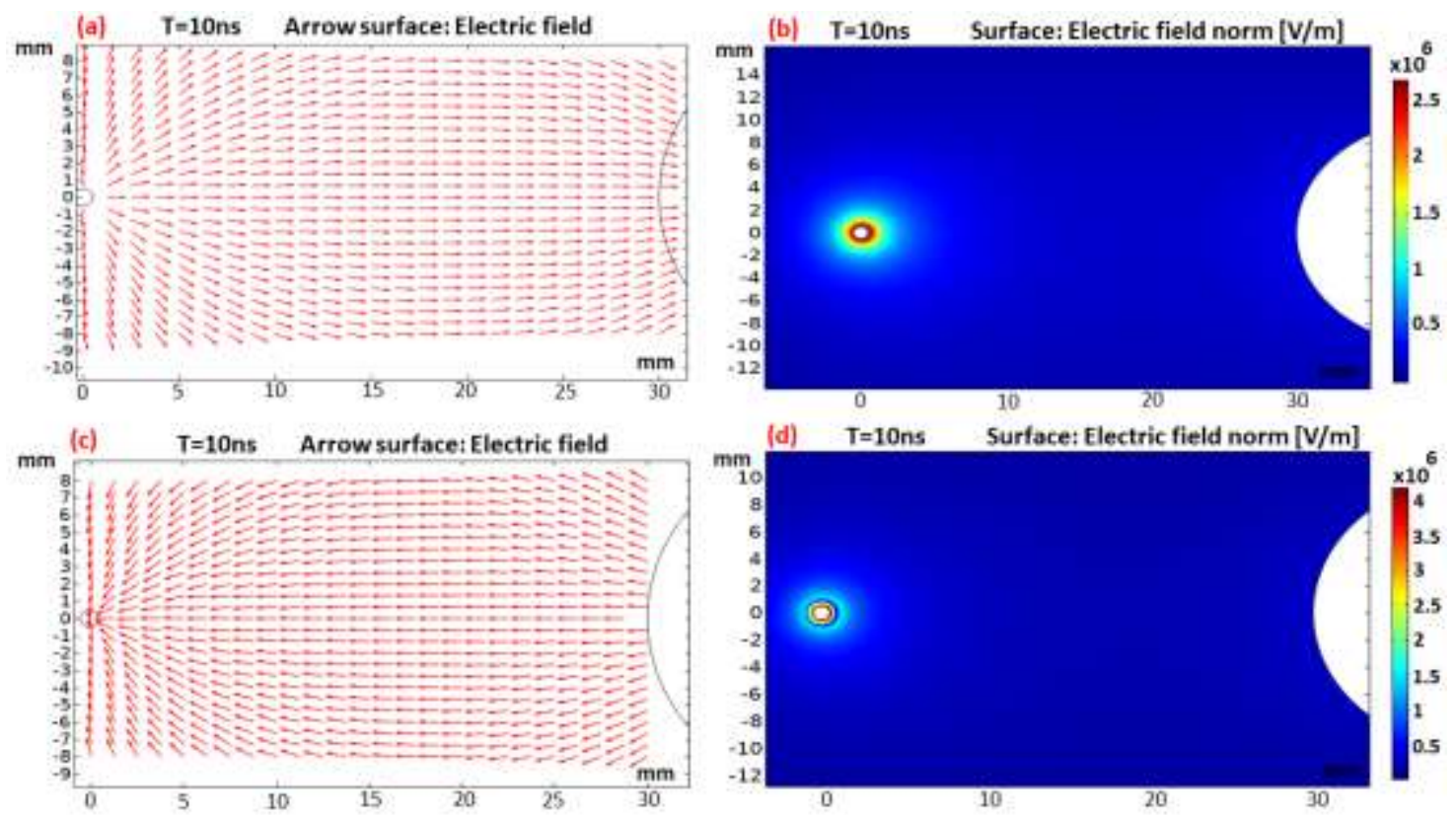

Figure 8. 2-D Plots of the electric field distributions (a) vector field (b) electric field magnitude in the positive corona discharge. (c) vector field, and (d) electric field magnitude for negative corona.

Figures 9 (a-e) and 10 (a-e) show the density distribution of charged species including $\mathrm{N}_{2}^{-}, \mathrm{O}_{2}^{-}$, $\mathrm{O}_{2}^{+}, \mathrm{N}_{2}^{+}$and electrons around the wire electrode in both positive and negative coronas, respectively. As can be seen in these, the electrical breakdown takes place only around the wire electrode with a length scale comparable to the wire diameter. The largest amount of $\mathrm{O}_{2}^{-}$ion in the positive and negative corona is $1.8 \times 10^{16} 1 / \mathrm{m}^{3}$ and $3.5 \times 10^{15} 1 / \mathrm{m}^{3}$, respectively, and that of for $\mathrm{N}_{2}^{+}$ion is $5.5 \times 10^{18} 1 / \mathrm{m}^{3}$ and $9 \times 10^{17} 1 / \mathrm{m}^{3}$, respectively. 


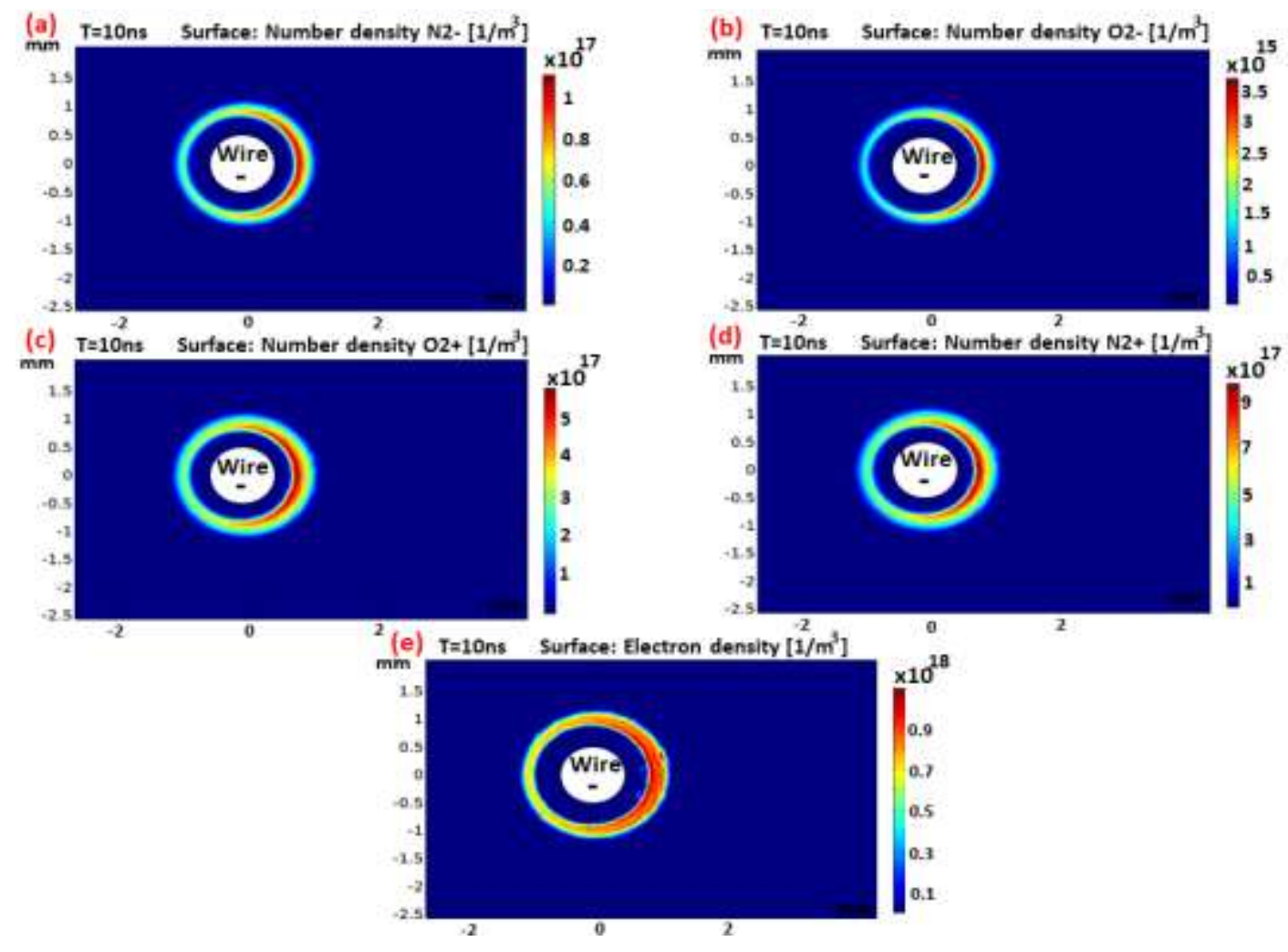

Figure 9. Species density distribution: (a) $\mathrm{N}_{2}^{-}$(b) $\mathrm{O}_{2}^{-}$(c) $\mathrm{O}_{2}^{+}$(d) $\mathrm{N}_{2}^{+}$and (e) electron in the negative corona discharge.
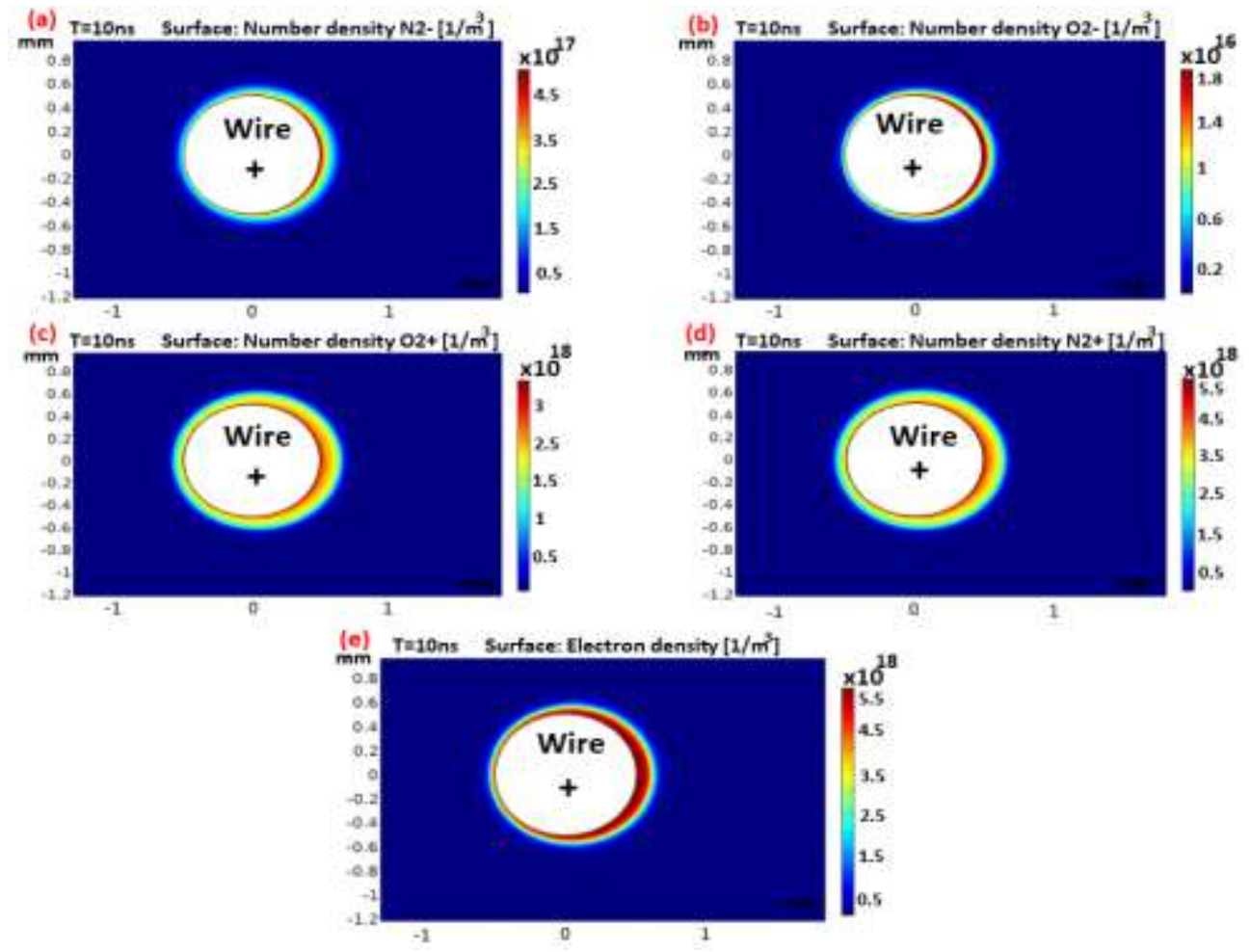
Figure 10. Species density distribution: (a) $\mathrm{N}_{2}^{-}$(b) $\mathrm{O}_{2}^{-}$(c) $\mathrm{O}_{2}^{+}$(d) $\mathrm{N}_{2}^{+}$and (e) electron in the positive corona discharge.

The magnitude and direction of the linear force were calculated according to equation (1). As can be seen in figures 11 (a) to (d), the linear term of the body force in the positive corona is a unidirectional vector in the whole gap between the electrodes as expected from theoretical considerations. While there is a zone whose linear force direction changes the sign in the negative corona. This can be understood by inspection of the product of the electric field $\mathrm{E}$ to the net space charge density $\rho$ in negative corona. That is, the direction of the linear term is towards the wire at the adjacent positions, outwards the wire in the space charge region, and finally, it is also towards the wire beneath the cylinder( figure 11 (c)). In positive corona discharge, the smoke from the incense flows rapidly from the wire to the cylinder by applying a high voltage. But in the negative corona condition, there occurs at first a turbulence flow of smoke from cylinder to wire, but after a very short time, the smoke flows from wire to cylinder-like that of the positive corona. The initial turbulence of flow could be attributed to the linear force action on the fluid.
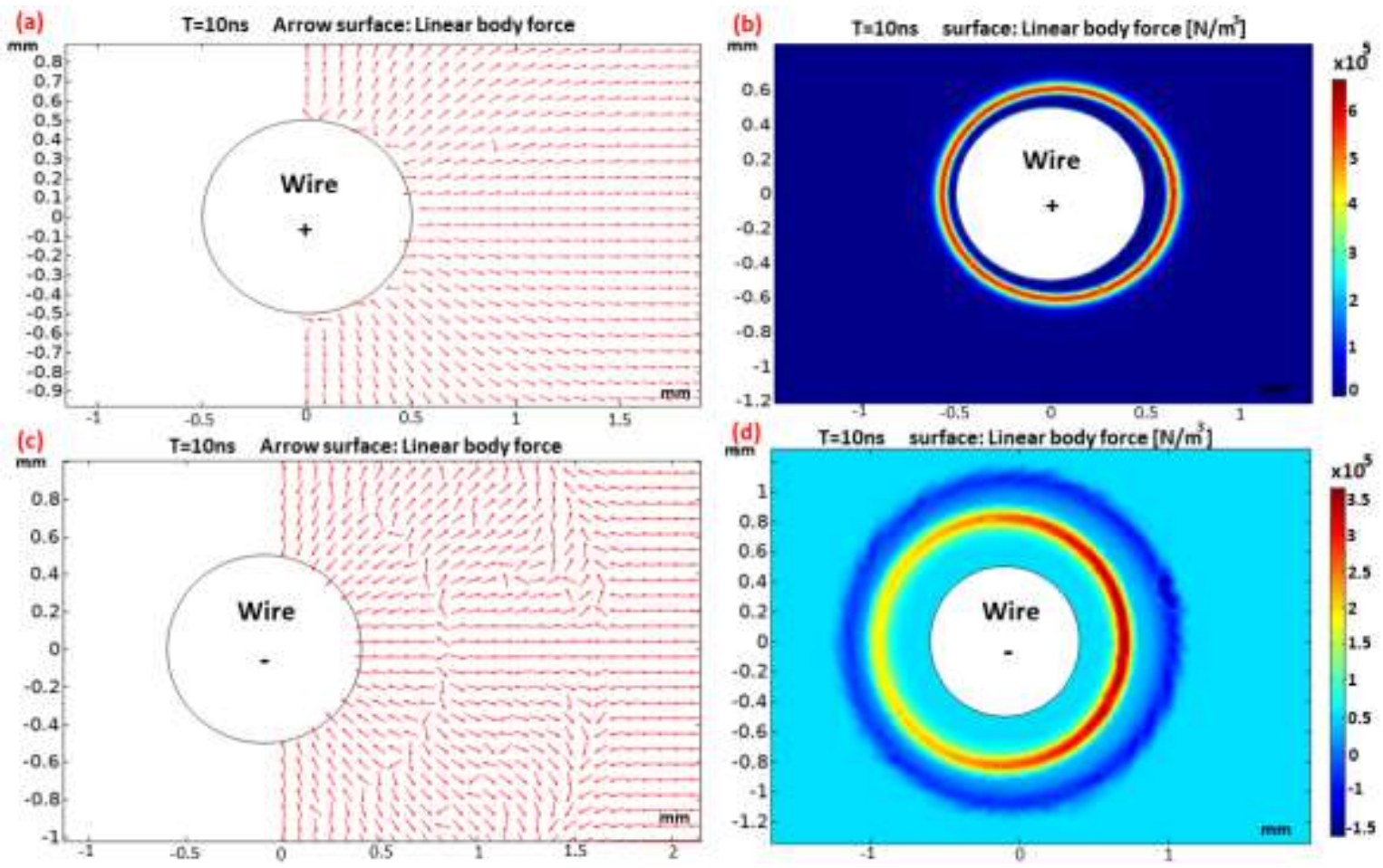

Figure 11. 2-D Plots of the linear body force term (a) vector field, (b) magnitude of the linear term in the positive corona discharge. (c) vector field, and (d) magnitude of the linear body force for negative corona. 
The magnitude and direction of the nonlinear body force were calculated using Equation (2). Figures 12(a), (b), (c), and (d) show that by changing the polarity of the applied voltage, the direction of the nonlinear force does not change. It points out to the cylinder electrode irrespective of the wire polarity. But the magnitude of the nonlinear force in the positive corona is larger than one order of magnitude than that of the negative corona. The nonlinear term was evaluated to be $7 \times 10^{11} \mathrm{~N} / \mathrm{m}^{3}$ and $2 \times 10^{10} \mathrm{~N} / \mathrm{m}^{3}$ for positive and negative corona, respectively. It is also worth noting that the magnitude of the nonlinear body force around the wire electrode is about 5 orders of magnitude higher than that of the linear force.
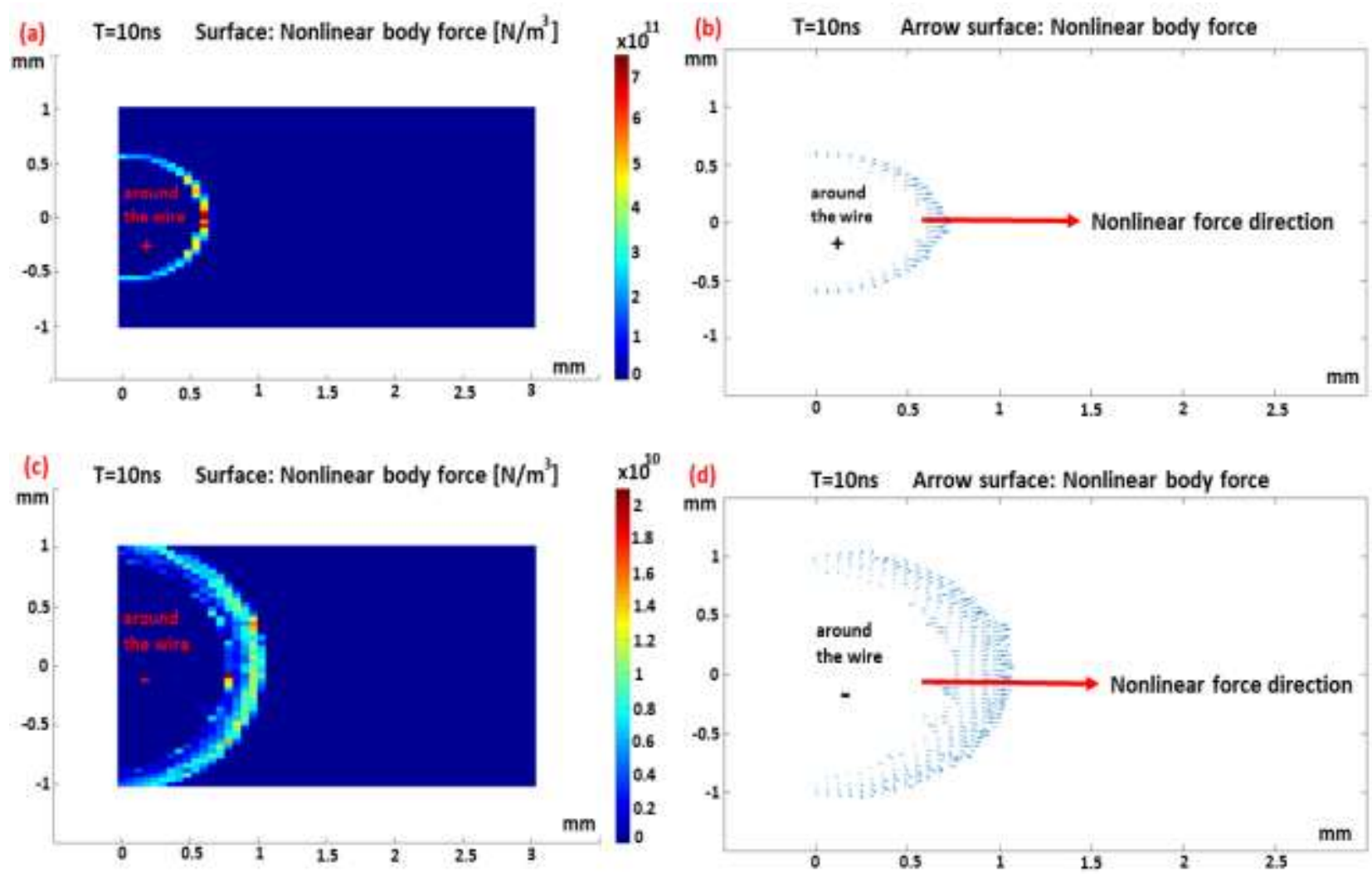

Figure 12. 2-D plots for the magnitude (a) and direction of the nonlinear body force (b) in the positive corona discharge. (c) magnitude, and (d) direction of the nonlinear force in the negative corona discharge. 

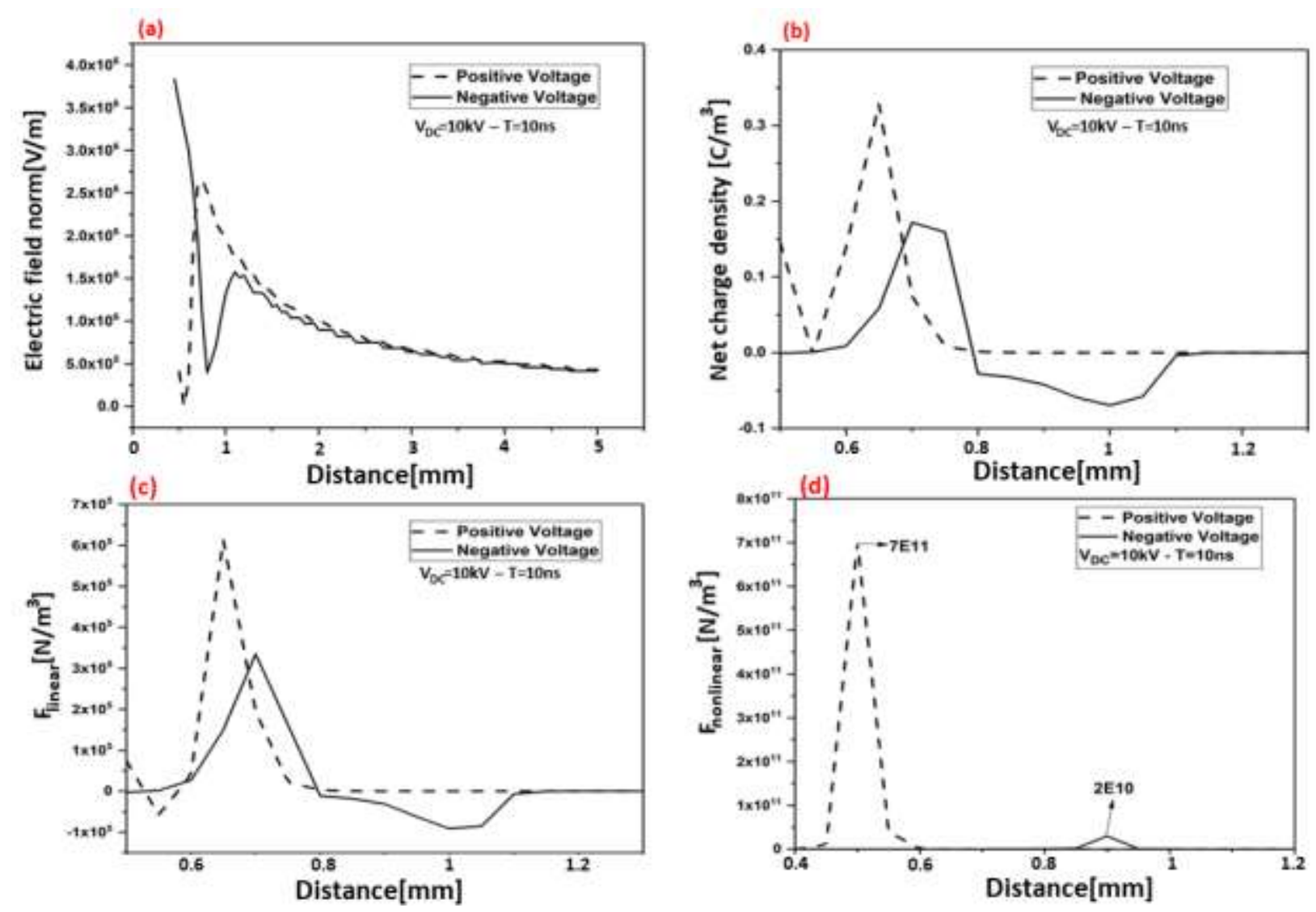

Figure 13. Displays, the electric field (a) net charge density (b) linear force (c) and nonlinear force (d) in positive and negative corona conditions according to cut line of figure 12.

The variations of the electric field strength, net charge density, linear and non-linear body force terms along with the line between the wire and the cylinder were depicted in figure 13. It is seen that there is a space charge region near the wire electrode as depicted in figure 13 (b). The space charge influences the linear body force and changes its direction between the points 0.6 to $0.8 \mathrm{~mm}$ for the negative corona(figure 13 (c)). This happens for the positive corona in a thin layer around the point of $0.55 \mathrm{~mm}$ near the wire. The most important insight into the electric thruster modeling is the evaluation of the nonlinear body force in positive and negative corona. As can be seen in figure 13(d), for positive corona, a strong nonlinear body force appears around the point of $0.5 \mathrm{~mm}$ where the electric field changes greatly (figures 13 (a)). In contrast to the positive corona, a less strong nonlinear body force is appeared on point of $0.9 \mathrm{~mm}$ in the negative corona. The variations in the strength of the linear and nonlinear body force terms together with their extensions were plotted in figure 14. It is seen that the nonlinear terms are 6 and 5 orders of magnitude larger than 
that of the linear terms in the positive and negative corona, respectively. Thanks to the strong gradient in the electric field near the wire.

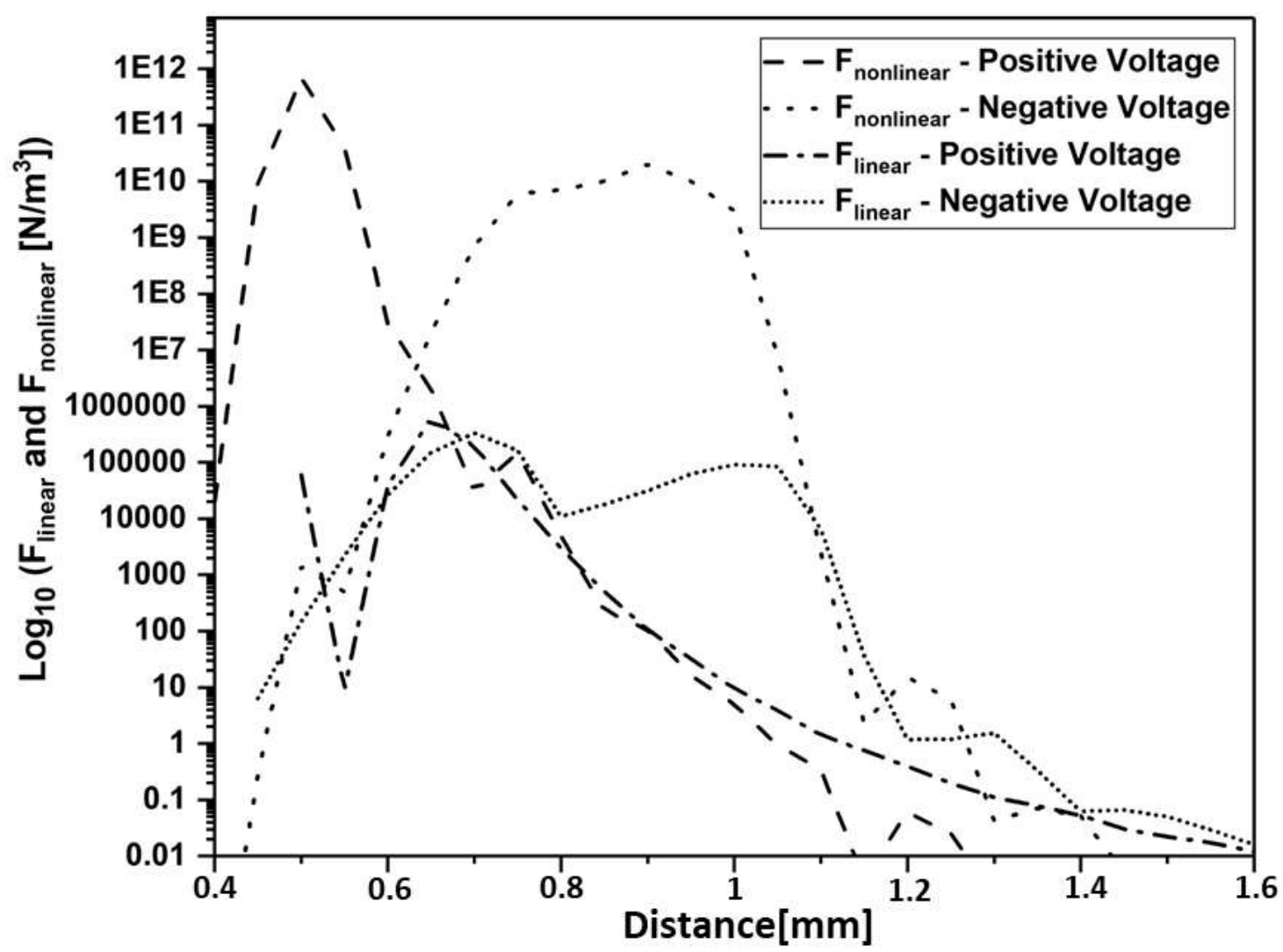

Figure 14. Plots of the linear and nonlinear body force terms around the wire at an applied voltage of $10 \mathrm{kV}$.

\subsection{Experimental Results}

In addition to the differences in thrust and electric wind between the positive and negative corona, there are some distinctions in feature and current characteristics. Figures 15 depicts the steadystate spatial distribution of the ignited corona around the wire for the positive (figure 15 a) and negative (figure $15 \mathrm{~b}$ ) corona at the same excitation voltage. The appearance of the corona in the positive and negative polarity are diffuse and contracted distributions, respectively. The positive corona manifested itself as a luminous layer around the wire electrode, while the negative one was bright beads. Although the corona assembly was supplied by the DC, the total current is intermittent constituted of both DC and AC terms flowing through the gap between the wire and the cylinder. Apart from the features of the corona, there is a significant difference in AC between the two coronas that was depicted in figures 15(a) and (b). Unlike the DC current, the AC current in the 
negative corona is larger than that of the positive one in all applied voltages. The dissimilarities were shown in figures 16 with taking into account the total current, $I_{t}=I_{D C}+I_{A C}$, too.

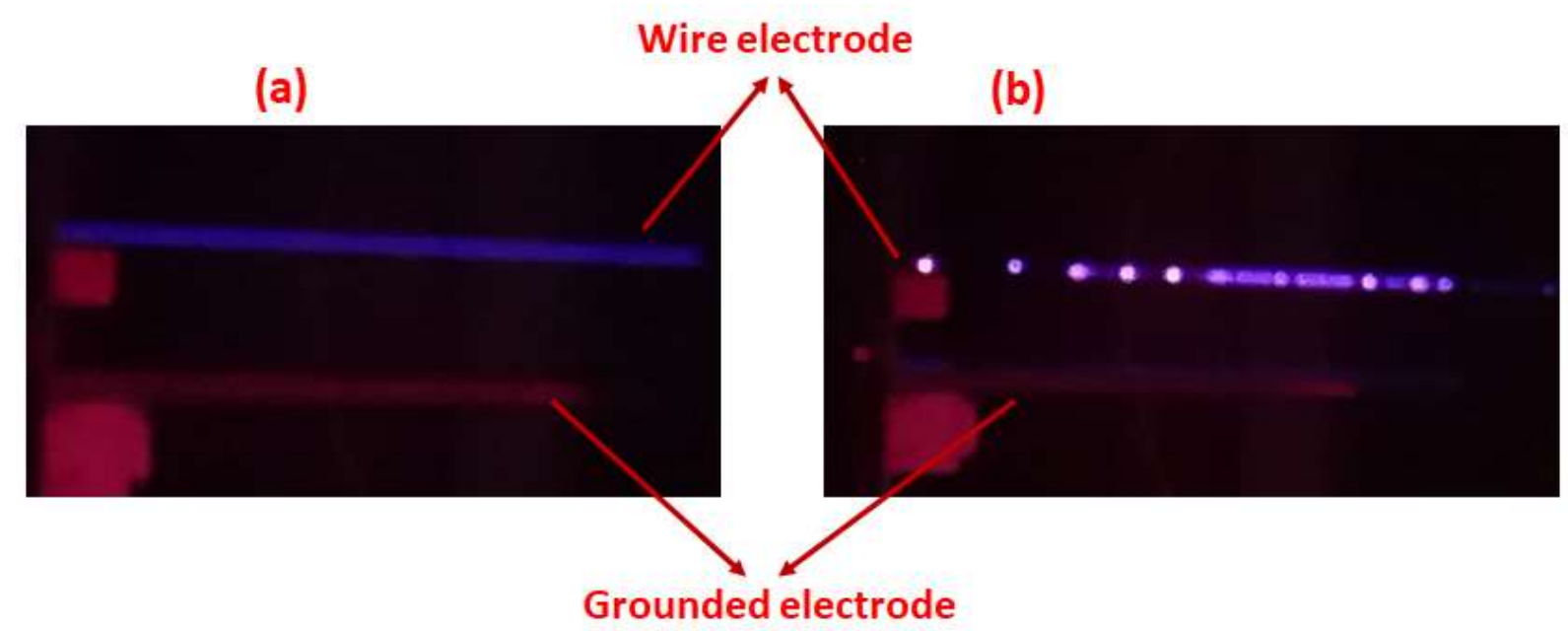

Figure 15. Spatial distribution of the corona around the wire, (a) positive corona discharge, and (b) negative corona

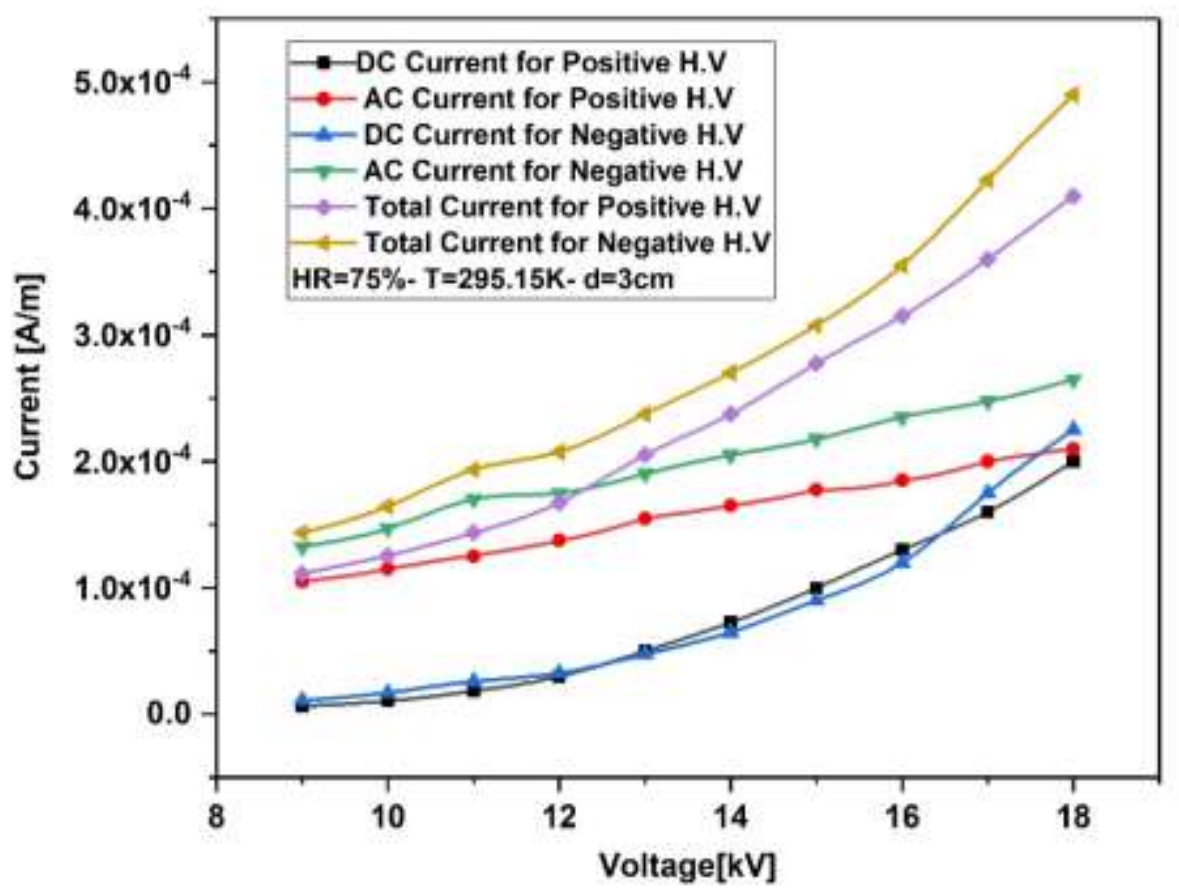

Figure 16. Changes in direct $\left(\mathrm{I}_{\mathrm{DC}}\right)$-pulse $\left(\mathrm{I}_{\mathrm{AC}}\right)$ and total $\left(\mathrm{I}_{\mathrm{DC}}+\mathrm{I}_{\mathrm{AC}}\right)$ electric currents versus positive and negative voltages

Zhang et al. showed that the Trichel pulse in a negative corona is a mode transition between low current Townsend discharge and high-current glow discharge [6]. The differences in the AC could be attributed to the Trichel pulse in negative corona. Figure 16 shows the voltage-current 
characteristic curve considering the total current $I_{\text {Total }}=I_{D C}+I_{A C}$ per unit spanwise length $(\mu \mathrm{A} / \mathrm{m})$. This may be due to the greater mobility of the negative ions than the positive ones in some conditions [41]. Despite a large AC current in the negative corona, the corresponding thrust is less than that of the positive corona. The evolutions of the body force were depicted in figure 17 versus the applied voltage (figure17 (a)), total current (figure 17 (b)), and the consumed power (figure 17 (c)). The trend of the thrust versus applied voltage differs little from that versus the power and total current. Up to $11 \mathrm{kV}$, the negative voltage has a larger thrust than the positive polarity, while beyond $11 \mathrm{kV}$, the positive corona has a large thrust.
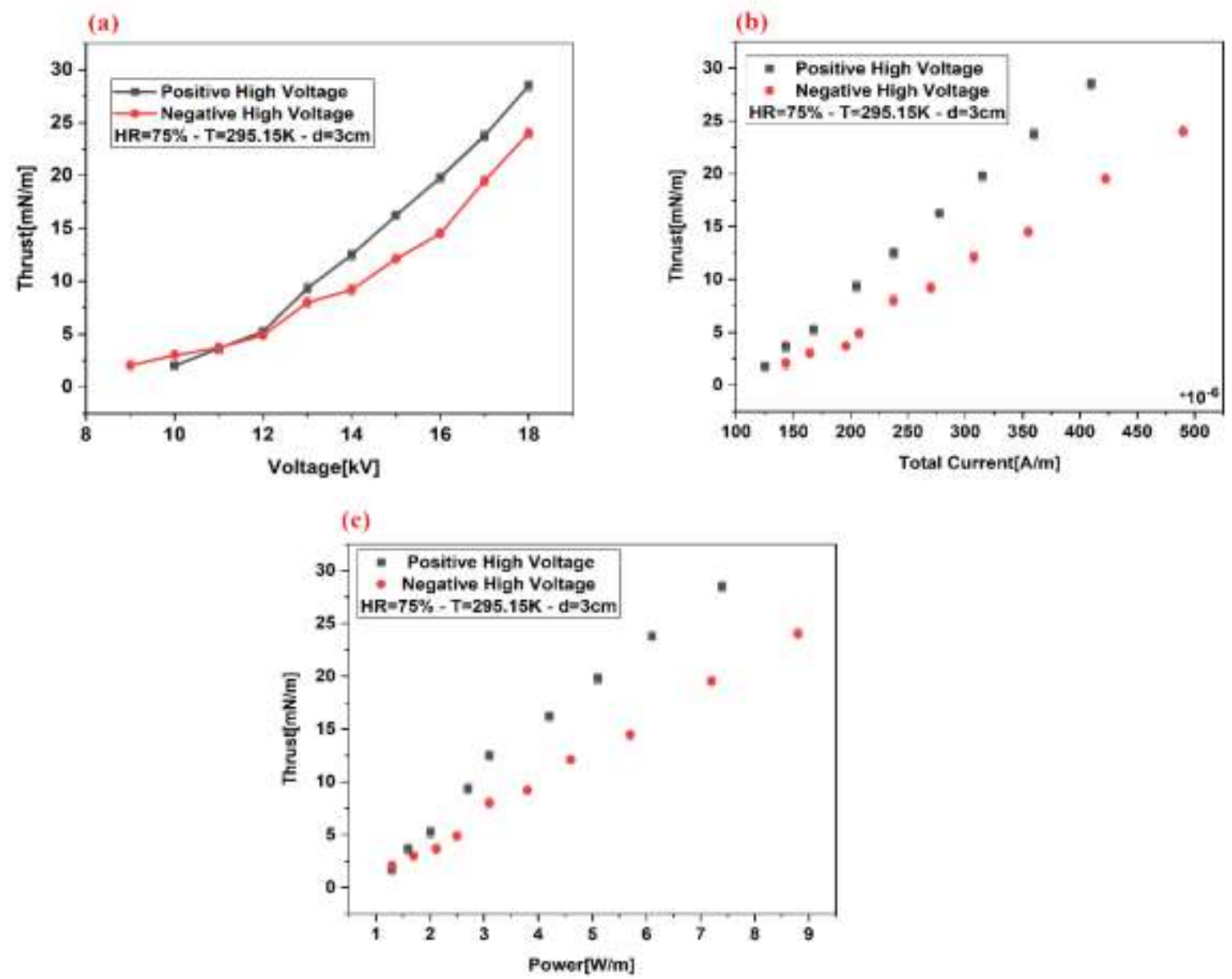

Figure 17. Thrust versus: (a) applied voltage (b) total electric current (c) consumed electrical power As the applied voltage increases, the density of the electrons and ions increases, and in turn, the momentum transfer to the neutrals grows, and eventually, thrust enlarges due to an increase in collisions. A similar trend happened for the effectiveness parameter of $\theta=T / P$ versus applied 
voltage for the thruster assemblies. At less than about $10.5 \mathrm{kV}$, the negative corona has a larger $\theta$, while beyond $10.5 \mathrm{kV}$, the positive corona plays more effectively as given in figure 18 .

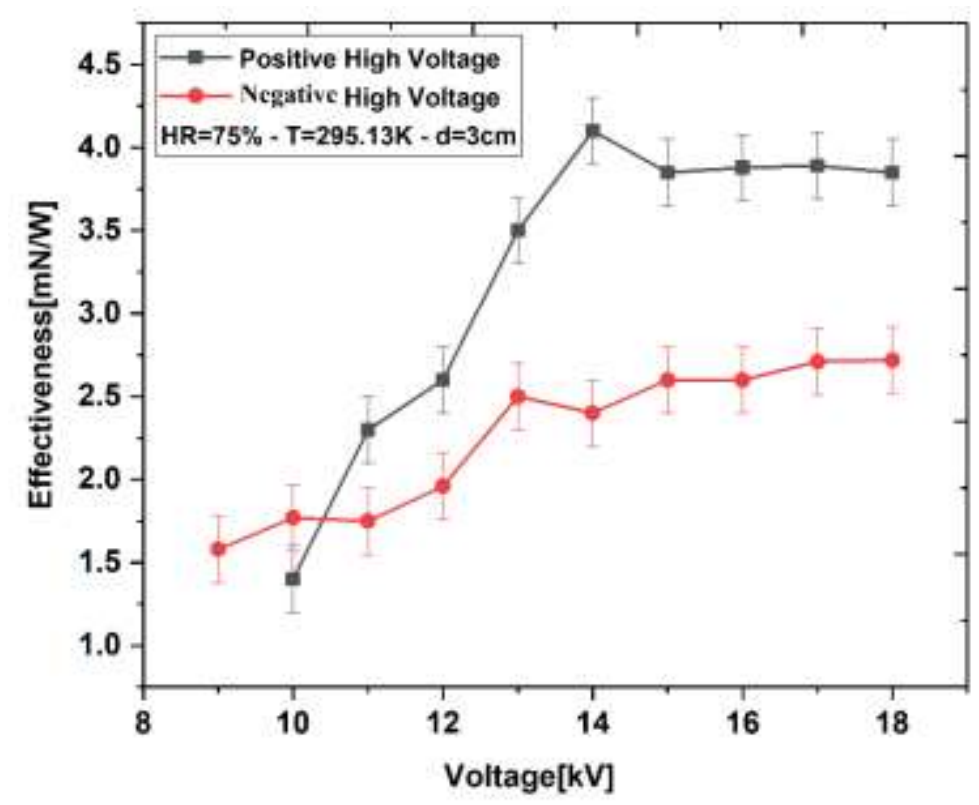

Figure 18. Effectiveness versus applied voltage in positive and negative corona.

The main outcome of the corona assembly could be addressed in the induced flow based on the electrohydrodynamic body forces. The electric flow trends could lead us to understand the physical concepts behind the corona thrusters. The evolutions in electric wind speed as a function of applied voltage and position were examined for both coronas. The results were given in figures 19 and 20, for positive and negative polarities, respectively. A glance at the results reveals that the trend of the induced electric wind for both structures is the same but it is efficient for the positive corona. The maximum speed of the induced flow were 2.4 and $1.7 \mathrm{~m} / \mathrm{s}$ for positive and negative corona, respectively, with the same applied voltage. An interesting aspect of the induced electric wind is its dependency on the distance from the powered electrode where the space charge plays a crucial effect. 


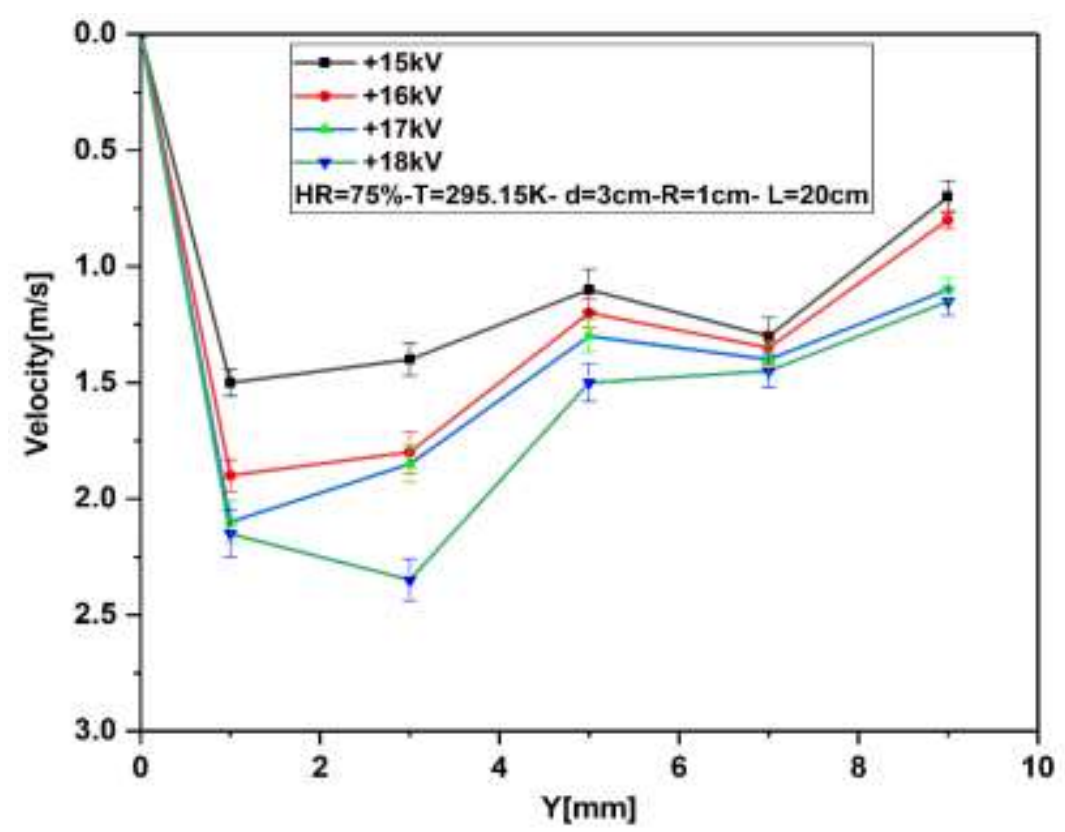

Figure 19. Velocity profiles for different positive voltage values.

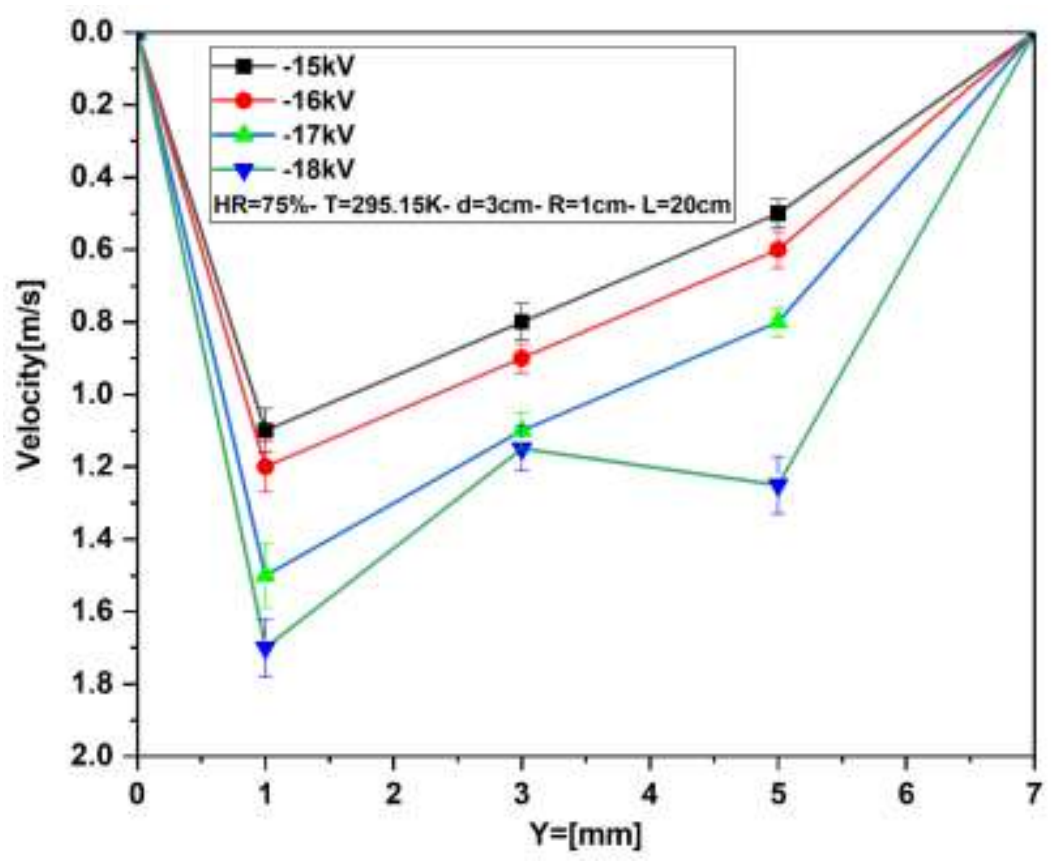

Figure 20. Velocity profiles for different negative voltage values.

To record the characteristics of the induced flow in both DC coronas, we employed smoke of the incense sticks between the wire and the cylinder. Before running the coronas, the smoke flow was upward, that is towards the wire (figure 21 (a)). As can be seen from Figures 21 (b) and (c), the appearance of the induced flow differs for the two types of coronas. For the positive corona, the induced wind looks like a laminar flow after the electrical excitation of the wire, but the induced 
wind resembles a turbulence flow for the negative corona at the initial excitation stage, eventually, it relaxes to a laminar flow. Another important characteristic of the electric wind is the initiation position which was proved to be beneath the wire. This result was depicted in figures 22(a) and (b) for positive corona with an applied voltage of $18 \mathrm{kV}$. The photos were taken with a few milliseconds seconds delay. Supplementary materials S1, S2, and S3 show short video clips on the induced electric wind feature corresponding to figures 21 and 22.
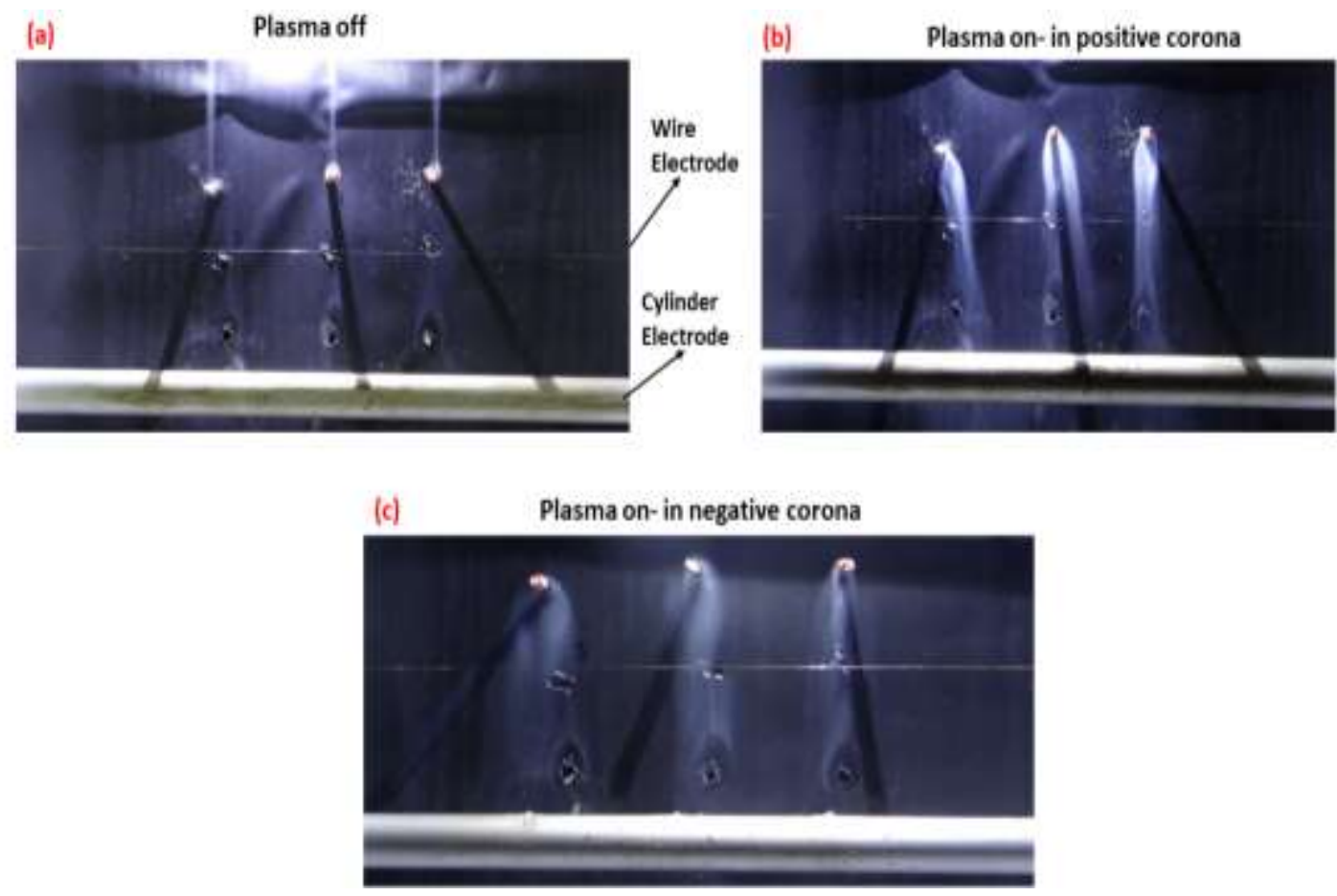

Figure 21. Changes in the direction of incense smoke movement: (a) corona-off (b) positive corona (c) negative corona at a voltage of $18 \mathrm{kV}$.

(a)
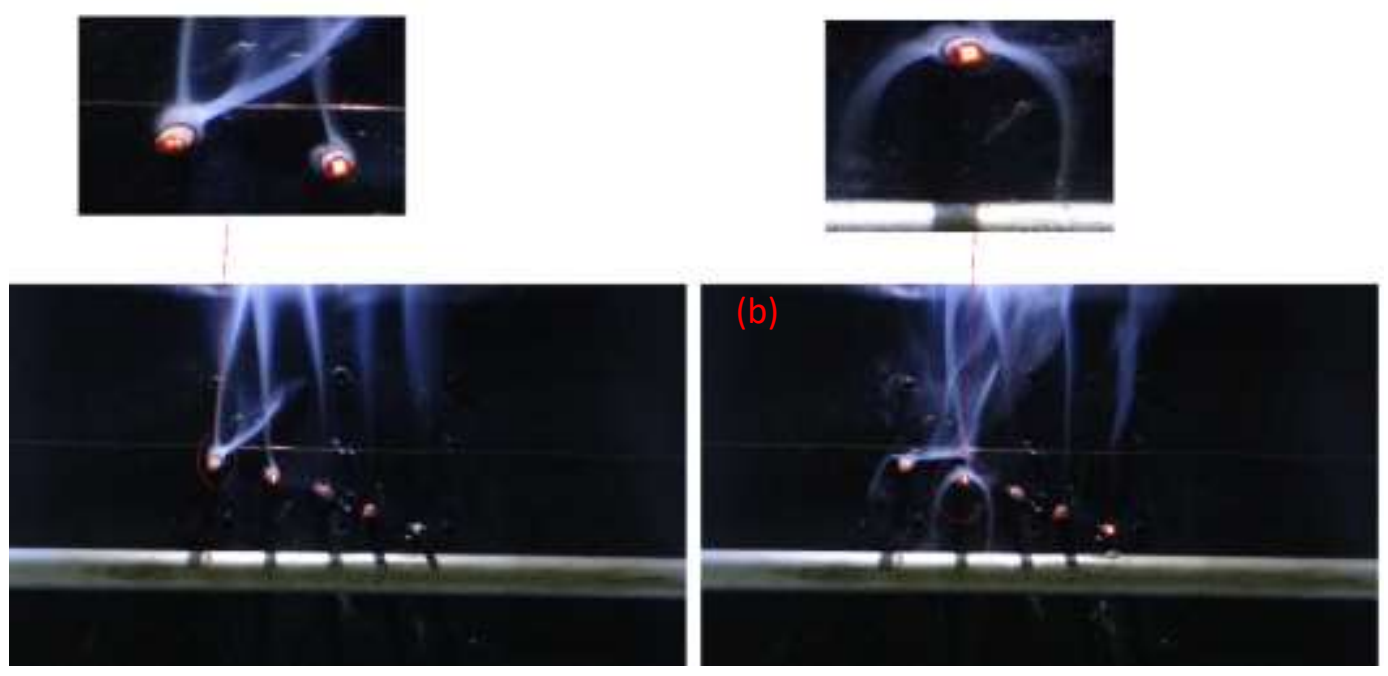

Figure22. Sequence change in the smoke movement: (a) at a voltage of $6 \mathrm{kV}$, initiation of the electric wind at the first incense position (b) at a voltage of $7 \mathrm{kV}$, the electric wind reaches the position of the second incense. 


\section{Corona thrusters perform like a pusher fan}

In this section, by assuming the induced flow to be incompressible, steady-state, and irrotational, we introduce a simple theory for the thrust evaluations using the variable mass equation. According to figures 23(a) and (b), it has been shown that the induced flow is generated at the wire periphery and extends towards the cylinder with an average speed of $\bar{V}$. The maximum velocity was created around the cylindrical electrode. Therefore, it can be assumed that the discharge assembly behaves like a variable mass system that generates the thrust via $T=d(m \bar{V}) / d t$, where $\mathrm{m}$ is the released mass of the system. Using the released mass of $m=\rho_{\text {air }} v$ in unit time and the corresponding volume $v=L D \bar{V}=S \bar{V}$, where, $v$ is the volume of the released flow in unit time, $L$ the length of the wire, $D$ the diameter of the cylinder, $\rho_{\text {air }}$ is the air density, and $\mathrm{S}$ is the cross-section of the cylinder. Then the thrust can be estimated as follows:

$$
T=\rho_{\text {air }} S \bar{V}^{2}
$$
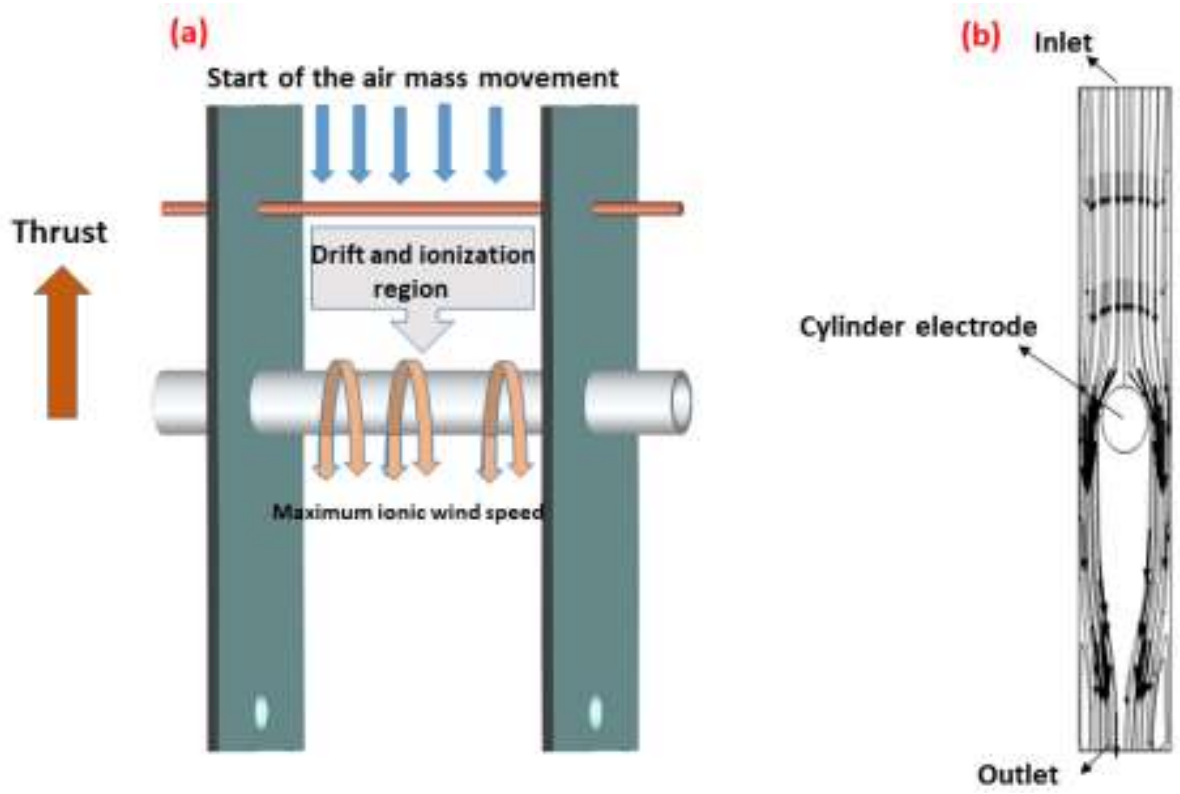

Figure 23. Displays, the mechanism of air mass movement (a) and velocity profiles around the cylinder (b).

Therefore, according to Equation 5, using the average electric wind speed around the cylinder electrode, the thrust force generated in a corona discharge can be calculated. The thrust direction is against the induced wind (upward) just like the thrust in a variable mass system. In another word, the corona thrusters play the role of a pusher fan on a boat irrespective of its polarity.

We used the measured velocity by Moreau et al to predict the thrust by equation (5). As can be seen in figure (24), there is a good agreement between our theoretical model and that measured by Moreau et al. 


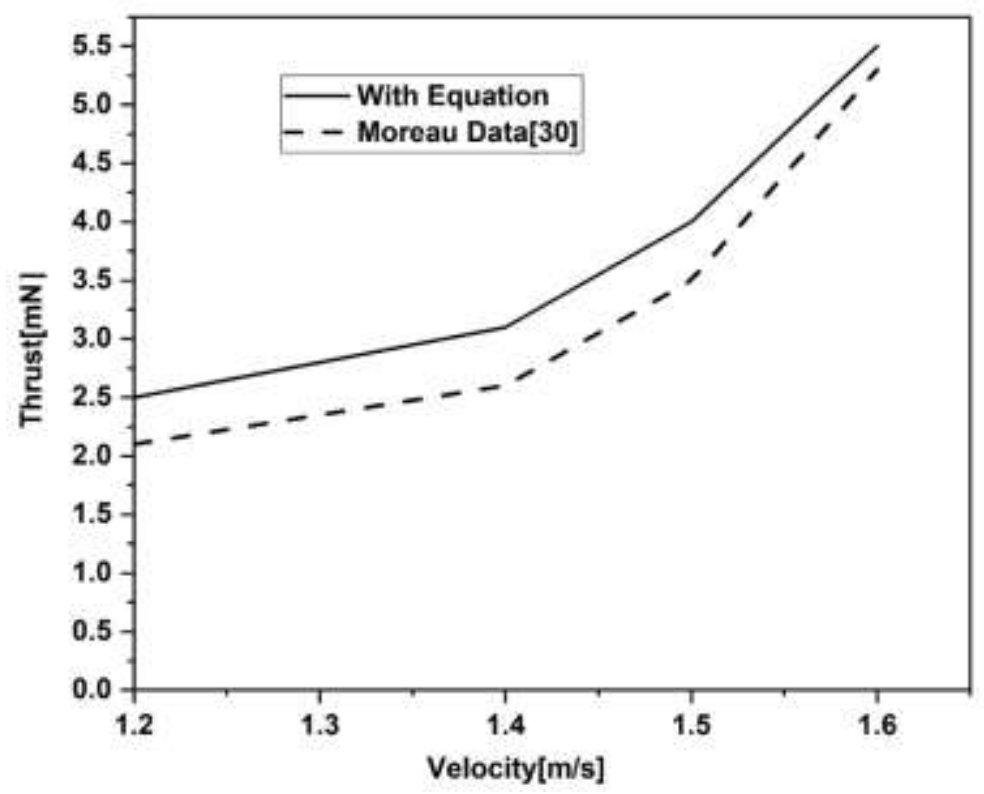

Figure 24. Applying equation (5) to the measured velocity by Moreau et al to predict the thrust. Solid and dashed lines are for equation (5) prediction and Moreau data, respectively.

Figures 25 and 26 show a comparison of the thrust obtained from Equation 5 based on the results of this paper in the positive and negative corona discharge, respectively.

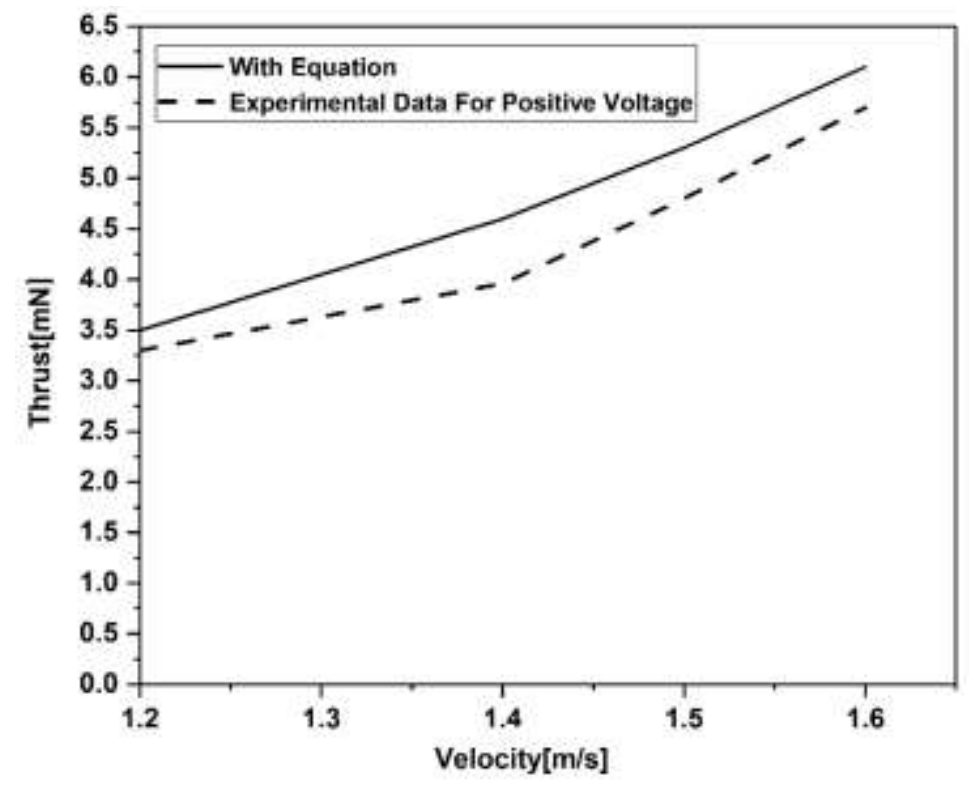

Figure 25. Comparison of the thrust force obtained from equation 5 with the report of this paper in positive corona 


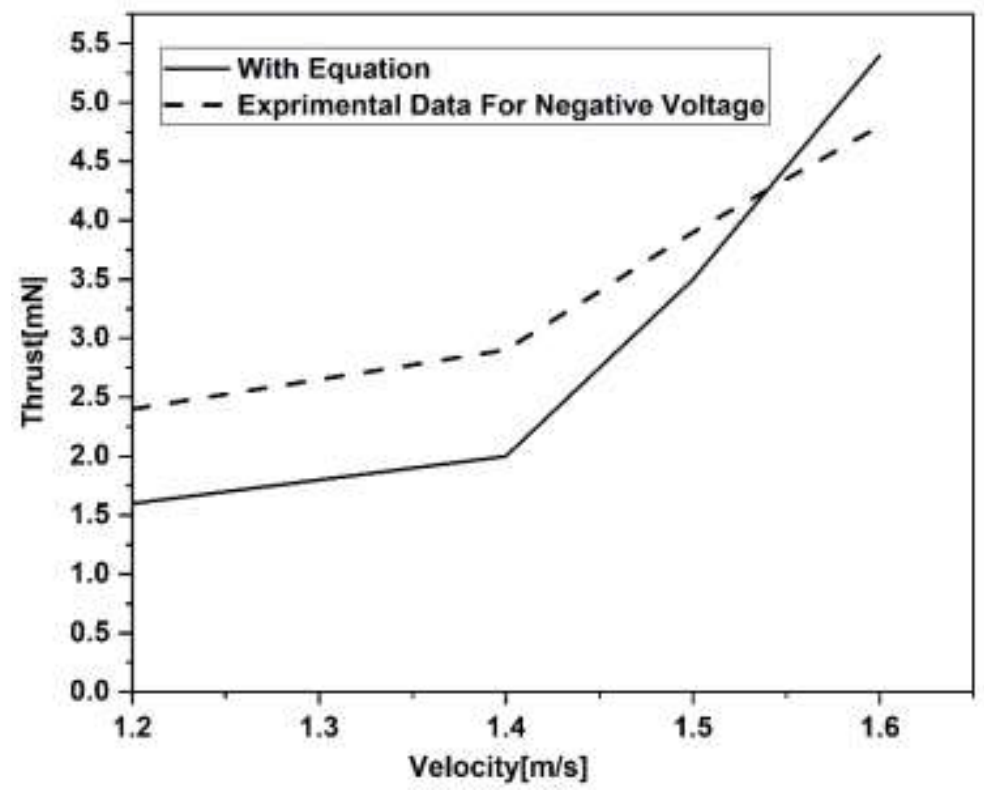

Figure 26. Comparison of the thrust force obtained from Equation 5 with the report of this paper in negative corona

In the case of the negative corona, the induced flow characteristic deviates from laminar flow, therefore, our assumptions do not satisfied with the released flow compared with that of the positive corona. As a result, there are deviations between the predicted and measured thrusts in the negative corona.

\section{Discussion}

As can be seen in figure 13(a), the corresponding external electric fields of the positive and negative coronas are screened by the space charge fields. In the positive corona, the screening effect takes place immediately after the wire around point 0.5 , then the field strength greatly increased and reaches its maximum at about $0.8 \mathrm{~mm}$ from the wire center. In this case, electrons are produced by ionization, then sink into the wire leading to a space charge of positive ions. The positive space charge is configured at two distinct layers separated by a neutral thin layer(figure 13 (b)). The first one attached to the wire possesses a small number of positive species relative to the second layer. The positive layers surrounding the wire create an electric field that is directed against the external field, then reduce the field around the wire, thus screening the external field. As the positive species travel to the ground(cathode) the strong field around the wire is restored. Then, a sudden increase of ionization follows, and an increase in current is observed. This is the reason for the intermittent current(AC) in the positive corona[1]. The electric current between the two adjacent peaks does not drop to zero, therefore we observe a DC component in the positive corona discharge. In contrast to the positive corona, the screening effect in the negative corona is less pronounced and it was happened around the point of $0.8 \mathrm{~mm}$, farther than the case of the positive one. For both coronas, the ionization zone is limited to the close distances to the wire whose electric field strength is considerable for ionization. The simulated space charges were depicted in figure 13(b) for both coronas. In the case of the negative corona, there is an apparent 
dipole constituted of negative and positive space charges. The induced dipole makes an electric field that is superimposed with the external one, as a result, the electric field is distorted in the gap. It is seen from figure 13(a), in the negative corona, the corresponding fields of the cathode and the induced dipole in the vicinity of the wire add up to give a field stronger than the positive corona. On the other hand, the resultant field inside the dipole zone is directed in the opposite direction that weakens the external field. The positive space charge locates close to the wire relative to the negative charge. The negative space charge rapidly flows to the anode, then the screening fails and the field strength enhances around the wire. In the atmospheric air, once the electrons travel towards the anode, they experience a weak electric field and attach to the electronegative molecules, forming negative ions like $\mathrm{O}_{2}^{-}$and $\mathrm{N}_{2}^{-}$. On the other hand, the positive ions flow to the wire leading to an increased current. In this case, the intermittent current is observed like that of the positive corona. Then the external field is restored and new ionization takes place.

Characteristics of the linear and nonlinear forces were depicted in figures 13(c) and (d) for both coronas. In the case of the positive corona, the direction of the linear force is almost towards the cathode parallel to the induced flow direction. It has a reversed direction with less amplitude in a small extension immediately after the wire, while it keeps the positive direction in the remaining gap. In the case of the negative corona, the direction of the linear force changes as a result of the induced dipole in the vicinity of the wire. The linear force direction is towards the anode after the wire while it reverses at the negative space charge location. The reversed linear force has a relatively large extension, that is, from $0.8 \mathrm{~mm}$ to $1.1 \mathrm{~mm}$ with a comparable amplitude with that of the former. Therefore, the resultant linear force weakens effectively the speed of the induced flow and makes turbulence on the flow movement. The orders of magnitude of linear force for both coronas are the same although their extensions and directions are different. Characteristics of the nonlinear force were depicted in figure 13(d). In contrast to the linear force, the nonlinear one has a unique direction from the wire towards the cylinder. The nonlinear force can be interpreted as a strong gradient in the intensity of the distorted electric field in the vicinity of the wire. So the magnitude of the nonlinear force strongly depends on the polarity of the corona which the space charges play a crucial role, as can be seen in figure 13(b). For the positive corona, the nonlinear force term is one order of magnitude larger than that of the negative corona. If the linear force term was the only responsible term for the induced flow and thrust, then the difference between the positive and negative corona must be very large, because the linear force term reverses in the corona bulk in the case of negative one. Meanwhile, the experiments revealed that both coronas led to the same flow direction with a little difference in the thrust magnitude. The direction and magnitude of the nonlinear force make the sense that it plays the main role in the wire-cylinder configuration thrusters. It provides unidirectional force independent of the corona polarity acting on both positive and negative species inside the corona medium. In the positive corona state, the nonlinear body force pushes back the electrons into the discharge channel and creates more ionization processes. With increasing ionization collisions, more thrust force and less pulsed electric current with irregular amplitude are created. The nonlinear force at the negative corona 
also causes a larger negative ion cloud to form around the cylindrical electrode. This creates a larger pulse current.

\section{Conclusions}

In this study, we provided new insight into electrostatic propulsion by introducing the nonlinear body force term affecting the induced flow in a wire-cylinder corona discharge configuration. The nonlinear body force arises from the strong gradient in electric field intensity independent of the corona polarity. If the linear force term was the only responsible term for the induced flow and thrust, then the difference between the positive and negative corona thrusts might be very large, because the linear force term reverses in the corona bulk in the case of negative discharge.

In the case of the positive corona, it was observed that the induced flow is almost laminar, but in the negative one, it is fairly chaotic due to the change in linear body force direction between the wire and the cylinder. Our simulations revealed that there takes place a strong screening in the external electric field in the negative corona discharge and an electric dipole originates near the wire electrode. The electric dipole is not observed in the positive corona, instead, a net positive space charge creates near the wire. The results of simulations indicated that the nonlinear body force is about 6 orders of magnitude larger than that of the linear one in positive corona. On the other hand, the nonlinear force term in the positive corona is one order of magnitude larger than that of the negative corona. The experimental results showed that both coronas led to the same flow direction with a little difference in the thrust magnitude. It was also shown that the corona thrusters are compatible with the variable mass systems, which the thrust value could be obtained from the released flow during the corona running. The thrust direction is against the induced flow direction like that of a pusher fan on a boat. The body force is responsible for induction flow in the corona assembly, and in turn, the released flow causes the thrust in the system.

\section{Acknowledgments}

The authors are grateful to Dr. Reza Ebrahimnezhad Darzi for providing valuable personal experiences in line with the analysis contained in this study.

\section{Reference}

[1]. Raizer, Y.P. \& Allen, J.E. Gas Discharge Physics. Springer, Berlin (1997).

[2]. Roth, J.R. Industrial Plasma Engineering. Nonthermal Plasma Processing (2001).

[3]. Akishev, Y. S., Dem'yanov, A. V., Karal'nik, V. B., Monich, A. E., \& Trushkin, N. I. Comparison of the AC barrier corona with DC positive and negative coronas and barrier discharge. Plas. Phys. Rep. 29, 82-91(2003).

[4]. Trichel, G. W. The mechanism of the positive point-to-plane corona in air at atmospheric pressure. Phys. Rev. 55, 382 (1939). 
[5]. Dordizadeh, P., Adamiak, K., \& Castle, G. P. Numerical investigation of the formation of Trichel pulses in a needle-plane geometry. App. Phys. 48, 415203 (2015).

[6]. He, B., Li, T., Xiu, Y., Zhao, H., Peng, Z., \& Meng, Y. Study on law of negative corona discharge in microparticle-air two-phase flow media. AIP. Adv. 6, 035114 (2016).

[7]. Zhang, Y., Qin, Y., Zhao, G., \& Ouyang, J. Time-resolved analysis and optical diagnostics of Trichel corona in atmospheric air. App. Phys. 49, 245206(2016).

[8]. Mizuno, A. Electrostatic precipitation. IEEE Transactions on Dielectrics and Electrical Insulation. 7, 615-624 (2000).

[9]. Locke, B. R., Sato, M., Sunka, P., Hoffmann, M. R., \& Chang, J. S. Electrohydraulic discharge and nonthermal plasma for water treatment. Industrial \& engineering chemistry research. 45, 882905 (2006).

[10]. Jaworek, A., Balachandran, W., Krupa, A., Kulon, J., \& Lackowski, M. Wet electroscrubbers for state of the art gas cleaning. Environmental science \& technology. 40, 6197-6207 (2006).

[11]. Van Durme, J., Dewulf, J., Leys, C., \& Van Langenhove, H. Combining non-thermal plasma with heterogeneous catalysis in waste gas treatment. A review. Applied Catalysis B: Environmental, 78, 324-333 (2008).

[12]. Kumar, S., Singh, R., Singh, T. P., \& Sethi, B. L. Surface modification by electrical discharge machining. A review. Journal of Materials Processing Technology, 209, 3675-3687 (2009).

[13]. Moreau, E. Airflow control by non-thermal plasma actuators. App. Phys. 40, 605 (2007).

[14]. Corke, T. C., Enloe, C. L., \& Wilkinson, S. P. Dielectric barrier discharge plasma actuators for flow control. Annual review of fluid mechanics, 42, 505-529 (2010).

[15]. Benard, N., \& Moreau, E. EHD force and electric wind produced by plasma actuators used for airflow control. In 6th AIAA Flow Control Conference (2012).

[16]. Brown, T. T. A method of and an apparatus or machine for producing force or motion. GB Patent, 300 (1928).

[17]. Bahder, T. B., \& Fazi, C. Force on an asymmetric capacitor. arXiv preprint physics.0211001 (2002).

[18]. Christenson, E. A., \& Moller, P. S. Ion-neutral propulsion in atmospheric media. AIAA journal. 5, 1768-1773 (1967).

[19]. Young, M., Keith, S., \& Pancotti, A. An overview of advanced concepts for near-space systems. In 45th AIAA/ASME/SAE/ASEE Joint Propulsion Conference (2009).

[20]. Moreau, E., Benard, N., Alicalapa, F., \& Douyère, A. Electrohydrodynamic force produced by a corona discharge between a wire active electrode and several cylinder electrodes-Application to electric propulsion. Journal of Electrostatics. 76, 194-200 (2015). 
[21]. Wilson, J., Perkins, H. D., \& Thompson, W. K. An investigation of ionic wind propulsion (2009).

[22]. Bondar, H., \& Bastien, F. Effect of neutral fluid velocity on direct conversion from electrical to fluid kinetic energy in an electro-fluid-dynamics (EFD) device. Journal of Physics D: App. Phys. 19, 1657 (1986).

[23]. Pekker, L., \& Young, M. Model of ideal electrohydrodynamic thruster. journal of propulsion and power. 27, 786-792 (2011).

[24]. Hauksbee, F. Physico-mechanical experiments on various subjects (No. 90). Johnson Reprint Corporation (1970).

[25]. Newton, I. Optics, pp. 25-27, London: Printers to the Royal Society, (1718).

[26]. Chattock, A. P. XLIV. On the velocity and mass of the ions in the electric wind in air. The London, Edinburgh, and Dublin Philosophical Magazine and Journal of Science. 48, 401-420 (1899).

[27]. Harney, D. J. An aerodynamic study of the" electric wind" (Doctoral dissertation, California Institute of Technology) (1957).

[28]. Davis, J. L., \& Hoburg, J. F. Wire-duct precipitator field and charge computation using finite element and characteristics methods. Journal of Electrostatics. 14, 187-199 (1983).

[29]. Jewell-Larsen, N. E., Karpov, S. V., Krichtafovitch, I. A., Jayanty, V., Hsu, C. P., \& Mamishev, A. V. Modeling of corona-induced electrohydrodynamic flow with COMSOL multiphysics. In Proc. ESA Annual Meeting on Electrostatics. (2008).

[30]. Colas, D. F., Ferret, A., Pai, D. Z., Lacoste, D. A., \& Laux, C. O. Ionic wind generation by a wire-cylinder-plate corona discharge in air at atmospheric pressure. Journal of applied physics. 108, 103306 (2010).

[31]. Moreau, E., Benard, N., Alicalapa, F., \& Douyère, A. Electrohydrodynamic force produced by a corona discharge between a wire active electrode and several cylinder electrodes-Application to electric propulsion. Journal of Electrostatics. 76, 194-200 (2015).

[32]. Moreau, E., Benard, N., Lan-Sun-Luk, J. D., \& Chabriat, J. P. Electrohydrodynamic force produced by a wire-to-cylinder dc corona discharge in air at atmospheric pressure. Journal of Physics D. App. Phys. 46, 475204 (2013).

[33]. Xu, H., He, Y., Strobel, K. L., Gilmore, C. K., Kelley, S. P., Hennick, C. C., ... \& Barrett, S. R. Flight of an aeroplane with solid-state propulsion. Nature. 563, 532-535 (2018).

[34]. Mahdavi, H., \& Sohbatzadeh, F. The role of nonlinear body force in production of electric wind in an asymmetric surface dielectric barrier discharge. Physica Scripta. 94, 085204 (2019).

[35]. Mehdipoor, M., Sohbatzadeh, F., \& Zabihpoor, M. Flow control with plasma-based actuator in different velocity regimes. Momentum and heat transfer effects. Contributions to Plasma Physics. 60, 201900037 (2020). 
[36]. Akishev, Y. S., Dem'yanov, A. V., Karal'nik, V. B., Monich, A. E., \& Trushkin, N. I. Comparison of the AC barrier corona with DC positive and negative coronas and barrier discharge. Plas. Phys. Rep. 29, 82-91(2003).

[37]. Hagelaar, G. H. M. BOLSIG+ Electron Boltzmann equation solver, (2010).

[38]. LXCat, electron scattering database, University of Toulouse, France, www.lxcat.net

[39]. Norberg, S. A., Johnsen, E., \& Kushner, M. J. Formation of reactive oxygen and nitrogen species by repetitive negatively pulsed helium atmospheric pressure plasma jets propagating into humid air. Plas. Sources. Sci. \& Tech. 24, 035026 (2015).

[40]. Shirafuji, T., \& Sawada, Y. Effects of ambient air on the characteristics of an atmosphericpressure plasma jet of a gas mixture of highly N2-diluted O2 on a sliding substrate. Japanese Journal of Applied Physics. 57, 01 AA06 (2017).

[41]. Bérard, P. Etude du vent ionique produit par décharge couronne à pression atmosphérique pour le contrôle d'écoulement aérodynamique (Doctoral dissertation, Ecole Centrale Paris) (2008). 


\section{Supplementary Files}

This is a list of supplementary files associated with this preprint. Click to download.

- S1.mp4

- S2.mp4

- S3.mp4

- Supplementaryvideo.docx 